Pure and Applied Mathematics Quarterly

Volume 10, Number 2

(Special Issue: In memory of

Andrey Todorov, Part 3 of 3)

$259-288,2014$

\title{
Local Holomorphic Curves on a Bounded Symmetric Domain in Its Harish-Chandra Realization Exiting at Regular Points of the Boundary
}

\section{Ngaiming Mok}

\begin{abstract}
We examine the asymptotic behavior of holomorphic curves on bounded symmetric domains in their Harish-Chandra realizations exiting at smooth points of the boundary, showing that such a holomorphic curve is aysmptotically totally geodesic at a generic point of its boundary and that furthermore the norm of the second fundamental form is bounded by a constant multiple of the Euclidean distance to the boundary.
\end{abstract}

Keywords: bounded symmetric domain, Harish-Chandra realization, second fundamental form, minimal disk, minimal rational tangent, maximal polydisk.

In the study of germs of holomorphic isometries up to normalizing constants in Mok [Mo5] between bounded domains with respect to the Bergman metric, denoted by $f:\left(\Omega_{1} ; x_{1}\right) \rightarrow\left(\Omega_{2} ; x_{2}\right)$, it was established that the graphs of such germs of holomorphic maps extend algebraically provided that the Bergman kernels $K_{\Omega_{i}}(z, w)$ are rational in $(z, \bar{w})$ for $i=1,2$. This applies especially to the case

Received February 8, 2013.

Research partially supported by the GRF grant 7046/10 of the Research Grants Council of Hong Kong, China. 
where $\Omega_{1} \Subset \mathbb{C}^{N_{1}}$ and $\Omega_{2} \Subset \mathbb{C}^{N_{2}}$ are bounded symmetric domains in their HarishChandra realizations, in which case it was also established that the algebraic extension of the germ $\operatorname{Graph}(f)$ restricts on $\Omega_{1} \times \Omega_{2}$ to the graph of a holomorphic isometric embedding. When $\Omega_{1}$ is irreducible and is of rank $\geq 2$ it follows from the proof of Hermitian metric rigidity in Mok [Mo1] that $f$ is necessarily totally geodesic, as observed in Clozel-Ullmo [CU]. It remains to understand the case where $\Omega_{1}$ is of rank 1 , i.e., $\Omega_{1}=\mathbb{B}^{n} \subset \mathbb{C}^{n}, n \geq 1$, is the complex unit ball. Restricting holomorphic isometries to complex 1-dimensional slices of $\mathbb{B}^{n}$ by complex affine lines, one obtains totally geodesic holomorphic curves which are isometric copies of the Poincaré disk, thus holomorphic isometries of the Poincaré disk into bounded symmetric domains are fundamental for the further study of holomorphic isometries between bounded symmetric domains. Non-standard examples of holomorphic isometric embeddings of the Poincaré disk into polydisks and Siegel upper half-planes were found in Mok [Mo5,§3, p.1647ff.], and a systematic study of the boundary behavior of arbitrary holomorphic isometric copies of the Poincaré disk on bounded symmetric domains was undertaken in Mok [Mo4] and Mok-Ng [MN1], where one makes use of structural equations concerning Gauss curvatures to study the asymptotic behavior of the second fundamental form as a variable point on the isometrically embedded Poincaré disk approaches a general boundary point.

While examining the asymptotic behavior of isometric copies of Poincaré disks, it was found that holomorphic curves defined on a neighborhood of a boundary point $b \in \partial \Omega$ of a bounded symmetric domain (in its Harish-Chandra realization) already exhibit interesting asymptotic properties. Since $\partial \Omega$ decomposes into strata under the action of $\operatorname{Aut}(\Omega)$, one has to study the asymptotic behavior depending on the stratum of $\partial \Omega$ where the boundary point $b$ lies. In this article we will study the asymptotic behavior in the simplest case, where the holomorphic curve on $\Omega$ concerned exits the bounded symmetric domain $\Omega$ at a smooth point of $\partial \Omega$. Let $D \Subset \mathbb{C}$ be the unit disk and denote by $b_{0} \in \partial D$ a boundary point. Let $U$ be an open neighborhood of $b_{0}$ on $\mathbb{C}$ of the form $U=D\left(b_{0} ; \epsilon\right)$ for some $\epsilon>0$, i.e., $U$ is the Euclidean disk of radius $\epsilon$ centered at $b_{0}$. Let $\Omega \Subset \mathbb{C}^{n}$ be a bounded symmetric domain in its Harish-Chandra realization, and $f: U \rightarrow \mathbb{C}^{N}$ be a holomorphic embedding such that $f(U \cap D) \subset \Omega$ and such 
that $f(U \cap \partial D) \subset \operatorname{Reg}(\partial \Omega)$, the smooth part of $\partial \Omega$. We will show that $f$ is asymptotically totally geodesic at $z \in U \cap D$ as the variable point $z$ approaches the boundary circle. We give a more precise estimate of the second fundamental form, and show that its norm decreases at least at the rate of the Euclidean distance $\delta$ to the boundary circle. In what follows $D \Subset \mathbb{C}$ is the unit disk and $\|\cdot\|$ denotes the norm on tensors naturally induced by the Kähler-Einstein metric $g$ on $\Omega$. Our principal result is the following Main Theorem.

Main Theorem. Let $\Omega \Subset \mathbb{C}^{N}$ be a bounded symmetric domain in its HarishChandra realization, and let $f: U \rightarrow \mathbb{C}^{N}$ be a holomorphic embedding such that $f(U \cap D) \subset \Omega$ and such that $f(U \cap \partial D) \subset \partial \Omega$. Suppose for any $b \in U \cap \partial D$, $f(b)$ is a smooth point of $\partial \Omega$. Denote by $\sigma(z)$ the second fundamental form at $z=f(w)$ of the (locally closed) complex submanifold $S:=f(U \cap D) \subset \Omega$ at $f(z)$ with respect to the Kähler-Einstein metric $g$ on $\Omega$. Then, for a general point $b \in U \cap \partial D$, the second fundamental form $\sigma(f(w))$ is asymptotically zero at $b$, i.e., $\lim _{w \in U \cap D, w \rightarrow b}\|\sigma(f(w))\|=0$. More precisely, for any neighborhood $U_{0}$ of $b$ in $\mathbb{C}^{N}$ such that $U_{0} \Subset U$, there is a positive constant $C$ depending on $U_{0}$ such that $\|\sigma(f(w))\| \leq C \delta(w)$ for any $w \in U_{0} \cap \Omega$.

\section{$\S 1$ Classification of irreducible bounded symmetric domains}

We start with a description of the classification of irreducible bounded symmetric domains $\Omega$. For more details we refer the reader to Wolf [Wo] and Mok [Mo2]. In what follows $M(p, q ; \mathbb{C})$ denotes the complex vector space of $p$-by- $q$ matrices $Z$ with complex coefficients, and $Z^{t}$ denotes the transpose of the matrix $Z$. The set of irreducible bounded symmetric domains breaks down into four classical series and two exceptional domains, as follows. We have

Irreducible Classical Symmetric Domains

$$
\begin{gathered}
D_{p, q}^{I}:=\left\{Z \in M(p, q ; \mathbb{C}): I-\bar{Z}^{t} Z>0\right\}, \quad p, q \geq 1 ; \\
D_{n}^{I I}:=\left\{Z \in D_{n, n}^{I}: Z^{t}=-Z\right\}, \quad n \geq 2 ; \\
D_{n}^{I I I}:=\left\{Z \in D_{n, n}^{I}: Z^{t}=Z\right\}, \quad n \geq 1 ; \\
D_{n}^{I V}:=\left\{\left(z_{1}, \ldots, z_{N}\right) \in \mathbb{C}^{N}:\|z\|^{2}<2 ;\|z\|^{2}<1+\left|\frac{1}{2} \sum_{i=1}^{N} z_{i}^{2}\right|^{2}\right\}, \quad n \geq 3 .
\end{gathered}
$$


$\underline{\text { Exceptional Domains }}$

$$
\begin{gathered}
D^{V}, \quad \text { of type } E_{6}, \quad \operatorname{dim}_{\mathbb{C}}\left(D^{V}\right)=16 ; \\
D^{V I}, \quad \text { of type } E_{7}, \quad \operatorname{dim}_{\mathbb{C}}\left(D^{V I}\right)=27 .
\end{gathered}
$$

The duplications in the listing are as follows: $D_{p, q}^{I} \cong D_{q, p}^{I} ; D_{1,1}^{I} \cong D_{2}^{I I} \cong D_{1}^{I I I} \cong$ $D$, the unit disk; $D_{3}^{I I} \cong D_{3,1}^{I} \cong \mathbb{B}^{3}$, the 3-dimensional unit ball; $D_{2}^{I I I} \cong D_{3}^{I V}$; $D_{2,2}^{I} \cong D_{4}^{I V}$ and $D_{4}^{I I} \cong D_{6}^{I V}$. The Harish-Chandra embedding (cf. Wolf [Wo], Mok [Mo2]) gives a canonical realization of every bounded symmetric domain as a bounded convex circular domain in a complex vector space. The description of the irreducible classical bounded symmetric domains $\Omega \Subset \mathbb{C}^{N}$ in the above gives precisely the Harish-Chandra realizations of these domains.

Denote by $G_{0}=\operatorname{Aut}_{0}(\Omega)$ the identity component of the automorphism group of $\Omega$. We say that $\Omega$ is of type $\mathfrak{g}$ if the Lie algebra of the complexification $G^{\mathbb{C}}$ of $G_{0}$ is of type $\mathfrak{g}$ according to the classification theory of simple complex Lie algebras in terms of Dynkin diagrams. The indices in the subscripts in the classical cases are subject to the same restrictions as in the above. We have

(1) $D_{p, q}^{I}$ is of type $A_{n}, n=p+q-1$;

(2) $D_{n}^{I I}$ is of type $D_{n}$;

(3) $D_{n}^{I I I}$ is of type $C_{n}$;

(4) $D_{n}^{I V}$ is of type $D_{k+1}$ when $n=2 k$ is even, and of type $B_{k+1}$ when $n=2 k+1$ is odd;

(5) $D^{V}$ is of type $E_{6}$;

(6) $D^{V I}$ is of type $E_{7}$.

\section{$\S 2$ The Kähler-Einstein metric along a minimal disk}

For the geometry of bounded symmetric domains, there is the important notion of maximal polydisks. We have

Polydisk Theorem (cf. Wolf [Wo, p.280]). Let $\Omega$ be a bounded symmetric domain of rank $r$, and $g$ be a Kähler metric on $\Omega$ invariant under the identity component $G_{0}=\operatorname{Aut}_{0}(\Omega)$ of the automorphism group Aut $(\Omega)$ of $\Omega$. Then, there exists an $r$-dimensional totally-geodesic complex submanifold $\Pi_{0}$ biholomorphic to the polydisk $D^{r}$. Moreover, $G_{0}$ acts transitively on the space of all such polydisks, and $\Omega=\bigcup\left\{\gamma \Pi_{0}: \gamma \in K\right\}$, where $K \subset G_{0}$ is the isotropy subgroup at $0 \in \Omega$. 
Any $\Pi:=\gamma \Pi_{0} \subset \Omega$ in the Polydisk Theorem is called a maximal polydisk of $\Omega$. Thus, any $\eta \in T(\Omega)$ is tangent to a maximal polydisk $\Pi \subset \Omega$.

For the purpose of studying holomorphic curves on bounded symmetric domains we will need to make use of calculations with respect to appropriate coordinate systems. For notational simplicity we will assume for the time being that $\Omega \Subset \mathbb{C}^{N}$ is an irreducible bounded symmetric domain, in which case any Kähler metric $g$ invariant under $G_{0}$ must already be Kähler-Einstein. Pick a maximal polydisk $\Pi \subset \Omega$ and denote by $H \subset G_{0}$ the Lie subgroup of automorphisms which fix $\Pi$ as a subset. Then, the restriction map $\rho: H \rightarrow \operatorname{Aut}(\Pi)$ is surjective (by Moore's Restricted Root Theorem, cf. Wolf [Wo]). In other words, $H$ restricts to the full automorphism group of the maximal polydisk $\Pi$. In particular, all factor disks of $\Pi \cong D^{r}$ are of constant Gaussian curvature $-\kappa$ for the same constant $\kappa>0$. By a minimal disk on $\Omega$ we mean a factor disk in any maximal polydisk $\Pi \subset \Omega$, noting that from the above $G_{0}$ acts transitively on the space of all minimal disks on $\Omega$. We normalize the choice of the canonical Kähler-Einstein metric $g$ so that minimal disks are of Gauss curvature -1 .

Let $K \subset G_{0}$ be the isotropy subgroup at $0 \in \Omega$. Write $G^{\mathbb{C}}$ for the complexification of $G_{0}, \mathfrak{g}^{\mathbb{C}}$ for the (complex) Lie algebra of $G^{\mathbb{C}}, \mathfrak{g}_{0} \subset \mathfrak{g}^{\mathbb{C}}$ for the (real) Lie algebra of $G_{0}$, which is a noncompact real form of $\mathfrak{g}^{\mathbb{C}}$, and $\mathfrak{k} \subset \mathfrak{g}_{0}$ for the Lie algebra of $K$. Fix a Cartan subalgebra $\mathfrak{h}$ of $\mathfrak{k}, \mathfrak{h}^{\mathbb{C}}:=\mathfrak{h} \otimes_{\mathbb{R}} \mathbb{C} \subset \mathfrak{k} \otimes_{\mathbb{R}} \mathbb{C}:=\mathfrak{k}^{\mathbb{C}} \cdot \mathfrak{h}^{\mathbb{C}} \subset \mathfrak{g}^{\mathbb{C}}$ being also a Cartan subalgebra of $\mathfrak{g}^{\mathbb{C}}$, we denote by $\Delta \subset \sqrt{-1} \mathfrak{h}^{\star}$ the set of all roots of $\mathfrak{g}^{\mathbb{C}}$, and by $\Delta_{0}^{+} \subset \Delta$ the subset of all positive noncompact roots. Let $\mu \in \Delta_{0}^{+}$ be the highest root, $\mathfrak{H}:=\{\rho \in \Delta: \mu-\rho \in \Delta\}$, and $\mathfrak{N}:=\left\{\rho \in \Delta_{0}^{+}: \mu-\rho \notin \Delta\right\}$. Write $p:=\operatorname{Card}(\mathfrak{H})$ and $q:=\operatorname{Card}(\mathfrak{N}), 1+p+q=N$. For a root $\varphi \in \Delta$ we denote by $\mathfrak{g}_{\varphi} \subset \mathfrak{g}^{\mathbb{C}}$ the root space associated to $\varphi$, i.e., the complex 1-dimensional space of root vectors belonging to $\varphi$. For a root $\rho \in \Delta_{0}^{+}$we will write $e_{\rho}$ for a vector belonging to $\rho$ of unit length with respect to the canonical Kähler-Einstein metric $g$, when we consider $\mathfrak{g}_{\rho} \subset \mathfrak{m}^{+}$and identify $\mathfrak{m}^{+}$canonically with $T_{0}(\Omega)$.

Let $P \subsetneq G^{\mathbb{C}}$ be the maximal parabolic subgroup of $G^{\mathbb{C}}$ containing $K$ (and hence its complexification $K^{\mathbb{C}}$ ). By the Borel embedding $\beta: \Omega \cong G_{0} / K \hookrightarrow$ $G^{\mathbb{C}} / P:=M$, the bounded symmetric domain $\Omega$ is realized as an open subset of its compact dual $M$, e.g., the type-I domain $D_{p, q}^{I}$ as an open subset of its compact dual $G(p, q)$, the Grassmannian manifold of $p$-planes in $\mathbb{C}^{p+q}$. The 
Harish-Chandra embedding gives an open embedding $\Omega \Subset \mathbb{C}^{N}$, where $\mathbb{C}^{N}$ can be canonically identified with a Zariski open subset of the compact dual $M$, giving $\Omega \Subset \mathbb{C}^{N} \subset M$. On the irreducible Hermitian symmetric manifold $M$ of the compact type a minimal rational curve is a rational curve of degree 1 with respect to the positive generator of $H^{2}(M, \mathbb{Z}) \cong \mathbb{Z}$. A non-zero $(1,0)$-vector tangent to a minimal rational curve is called a minimal rational tangent. For the highest root $\mu \in \Delta_{0}^{+}$, the root spaces $\mathfrak{g}_{\mu}$ resp. $\mathfrak{g}_{-\mu}$ generate a 3 -dimensional complex Lie algebra $\mathfrak{g}^{\mathbb{C}}[\mu]=\mathfrak{g}_{\mu}+\mathfrak{g}_{-\mu}+\left[\mathfrak{g}_{\mu}, \mathfrak{g}_{-\mu}\right]$ isomorphic to $\mathfrak{s l}(2, \mathbb{C})$ corresponding to a Lie subgroup $G^{\mathbb{C}}[\mu] \subset G^{\mathbb{C}}$ such that $G^{\mathbb{C}}[\mu] \cong \mathbb{P S L}(2, \mathbb{C})$ and such that the $G^{\mathbb{C}}[\mu]$-orbit $C$ of $0=e P \in M$ is a minimal rational curve on $M$. The intersection $C \cap \Omega$ is the orbit of a real form $G_{0}[\mu] \subset G^{\mathbb{C}}[\mu]$ such that $G_{0}[\mu]$ is isomorphic to $\mathbb{P S U}(1,1)$. Moreover, $C \cap \Omega=G_{0}[\mu] \cdot 0$ is a minimal disk on $\Omega$. For a general reference cf. Wolf [Wo]. Regarding tangents to minimal disks we have the following result from Mok [Mo1].

Lemma 1. Let $\Omega \Subset \mathbb{C}^{N} \subset M$ be the Harish-Chandra realization $\Omega \Subset \mathbb{C}^{N}$ of an irreducible bounded symmetric domain $\Omega$ together with the Borel embedding $\Omega \subset M$ into its dual Hermitian symmetric space $M$ of the compact type. Let $\mathcal{S} \subset \mathbb{P} T(\Omega)$ be the subset consisting of projectivizations of non-zero vectors $\eta$ tangent to minimal disks, and $\mathcal{C} \subset \mathbb{P} T(M)$ be the set of projectivizations of minimal rational tangents. Then $\mathcal{S}=\mathcal{C} \cap \mathbb{P} T(\Omega)$. As a consequence, denoting by $\mathcal{S}_{x} \subset \mathbb{P} T_{x}(\Omega)$ the fiber of $\pi: \mathcal{S} \rightarrow \Omega$ over $x \in \Omega$, in terms of trivializations of $\mathbb{P} T(\Omega)$ given by Harish-Chandra coordinates we have $\mathcal{S}=\mathcal{S}_{0} \times \Omega \subset \mathbb{P} T_{0}(\Omega) \times \Omega=$ $\mathbb{P} T(\Omega)$.

We note that the last statement follows from $\mathcal{S}=\mathcal{C} \cap \mathbb{P} T(\Omega)$. In fact, $\mathcal{S} \subset \mathbb{P} T(M)$ is invariant under $G^{\mathbb{C}}$, the identity component of the automorphism group of $M$, hence it is in particular invariant under the abelian Lie subgroup $\mathfrak{M}^{+}:=\exp \left(\mathfrak{m}^{+}\right) \subset G^{\mathbb{C}}$ corresponding to the abelian subalgebra $\mathfrak{m}^{+} \subset \mathfrak{g}^{\mathbb{C}}$ in the Harish-Chandra decomposition $\mathfrak{g}^{\mathbb{C}}=\mathfrak{m}^{+} \oplus \mathfrak{k}^{\mathbb{C}} \oplus \mathfrak{m}^{-}$, where $\mathfrak{m}^{+}$is the direct sum of $\mathfrak{g}_{\rho}, \rho \in \Delta_{0}^{+}$, and $\mathfrak{m}^{-}=\overline{\mathfrak{m}^{+}}$. From the construction of the HarishChandra embedding $\mathfrak{M}^{+} \subset G^{\mathbb{C}}$ preserves $\mathbb{C}^{N}$, and $\mathfrak{M}^{+}$acts as the group of Euclidean translations when restricted to $\mathbb{C}^{N}$. Thus, from $\mathcal{S}=\mathcal{C} \cap \mathbb{P} T(\Omega)$ it follows that the fiber $\mathcal{S}_{x}$ of $\mathcal{S}$ over $x \in \Omega$ is identified with $\mathcal{S}_{0}$ via the Euclidean translation $\tau_{x}: \mathbb{C}^{N} \rightarrow \mathbb{C}^{N}$ defined by $\tau_{x}(z)=z+x$. We denote by $\widetilde{\tau}_{x} \in G^{\mathbb{C}}$ 
the unique automorphism which restricts to $\tau_{x}$ on $\mathbb{C}^{N}$. Noting that the minimal rational curves $C$ passing through $0 \in \Omega \subset M$ are compactifications of complex 1-dimensional linear subspaces $\mathbb{C} \eta,[\eta] \in \mathcal{S}_{0}$, we write $C=C_{\eta}$. For $x \in \mathbb{C}^{N}$, a minimal rational curve $C^{\prime}$ passing through $x$ is the closure of a complex 1dimensional affine line $L \subset \mathbb{C}^{N}$. Thus $L=\tau_{x}(\mathbb{C} \eta)=\mathbb{C} \eta+x$, and $C^{\prime}=\widetilde{\tau}_{x}\left(C_{\eta}\right)=$ $\overline{\tau_{x}(\mathbb{C} \eta)}=\bar{L}=\overline{\mathbb{C} \eta+x} \subset M$. In particular, for $x \in \Omega$, minimal disks on $\Omega$ passing through $x$ are of the form $L \cap \Omega$ for a certain class of complex 1-dimensional affine linear subspaces $L \subset \mathbb{C}^{N}$ passing through the point.

Making use of orthonormal bases consisting of root vectors as in the above we have the following preparatory lemma about the expression of the KählerEinstein metric $g$ along a minimal disk.

Lemma 2. Choose an orthonormal basis $\left\{e_{k}\right\}_{k=1}^{N}$ of $T_{0}(\Omega) \cong \mathbb{C}^{N}$ consisting of root vectors of unit length such that $e_{1}$ belongs to the highest root $\mu, e_{k}$ belongs to some root $\rho \in \mathfrak{H}$ for $2 \leq k \leq p+1$, and $e_{\ell}$ belongs to some root $\rho \in \mathfrak{N}$ for $p+2 \leq \ell \leq N$. Then, $e_{1}$ is a minimal rational tangent at 0 . Moreover, writing $D_{0}:=\mathbb{C} e_{1} \cap \Omega$ for the minimal disk tangent to $e_{1}$ and expressing in terms of the Euclidean coordinates $\left(z_{1}, \cdots, z_{N}\right)$ corresponding to the choice of orthonormal basis $\left\{e_{k}\right\}_{k=1}^{N}$, for any point $z \in D_{0}$, the matrix $\left(g_{i \bar{j}}(z)\right)_{i, j=1}^{N}$ representing the Kähler-Einstein metric $g$ at $z \in D_{0}$ is a diagonal matrix.

Proof. The fact that $e_{1}$ is a minimal rational tangent and $D_{0} \subset \Omega$ is a minimal disk is in Wolf [Wo] (cf. Lemma 1 here and the paragraph following it). Let $H \in \mathfrak{h}$ and consider the real 1-parameter subgroup $\exp (\mathbb{R} H) \subset \exp (\mathfrak{h}) \subset K$. The 1-parameter group $\exp (\mathbb{R} H)$ acts as a group of unitary transformations on $\mathbb{C}^{N} \cong T_{0}(\Omega)$ fixing $\Omega$ as a set, and we will use the same notation to denote both the unitary transformations and the automorphisms of $\Omega$ which are the restrictions of the unitary transformations to $\Omega$. We also write $e^{t H}$ for $\exp (t H)$. Recall that the set of all roots $\Delta$ lie in $\sqrt{-1} \mathfrak{h}^{*}$. For any $\rho \in \Delta_{0}^{+}, \mathfrak{g}_{\rho}$ is an eigenspace of $\operatorname{ad}(H)$, and, writing $H^{\prime}=-\sqrt{-1} H$, we have $e^{t H}\left(v_{\rho}\right)=e^{\rho\left(H^{\prime}\right) i t} v_{\rho}$ for any $v_{\rho} \in \mathfrak{g}_{\rho}$, noting that $\rho\left(H^{\prime}\right)=-\sqrt{-1} \rho(H) \in \mathbb{R}$ since $\Delta \subset \sqrt{-1} \mathfrak{h}^{*}$. If $H \in \mathfrak{h}$ such that $\mu(H)=0$, then $e^{t H}\left(v_{\mu}\right)=v_{\mu}$ for any $v_{\mu} \in \mathfrak{g}_{\mu}$. In particular, $\exp (t H)$ fixes every point $z$ on the minimal disk $D_{0}=\mathbb{C} e_{1} \cap \Omega$. Since $\exp (\mathfrak{h}) \subset K$ acts as a group of isometries on $\Omega$, for any $x \in D_{0}$ and for any $\xi, \eta \in T_{x}(\Omega)$, we must have $g(\xi, \bar{\eta})=g\left(e^{t H} \xi, \overline{e^{t H} \eta}\right)$. Let now $\rho, \tau \in \Delta_{0}^{+}$be distinct positive 
noncompact roots. If now we choose $\xi=e_{\rho}=e_{i}$ and $\eta=e_{\tau}=e_{j}$, at $x \in D_{0}$ we must have

$$
\begin{aligned}
g_{i \bar{j}}(x) & =g\left(e_{\rho}, \overline{e_{\tau}}\right)=g\left(e^{t H} e_{\rho}, \overline{e^{t H} e_{\tau}}\right)=g\left(e^{\rho\left(H^{\prime}\right) i t} e_{\rho}, \overline{e^{\tau\left(H^{\prime}\right) i t} e_{\tau}}\right) \\
& =e^{\left(\rho\left(H^{\prime}\right)-\tau\left(H^{\prime}\right)\right) i t} g\left(e_{\rho}, \overline{e_{\tau}}\right)=e^{\left(\rho\left(H^{\prime}\right)-\tau\left(H^{\prime}\right)\right) i t} g_{i \bar{j}}(x),
\end{aligned}
$$

for every $t \in \mathbb{R}$, and we will be able to complete the proof of Lemma 1 if we can verify

$(\sharp)$ Given any $\rho, \tau \in \Delta_{0}^{+}$there exists $H \in \mathfrak{h}$ such that $\mu(H)=0$ and $\rho(H) \neq \tau(H)$,

since $(\sharp)$ obviously implies that $g_{i \bar{j}}(x)=0$ for every $x \in D_{0}$ and for $i \neq j$. To verify ( $\sharp$ ) note first of all that there exists a central element $H_{0} \in \sqrt{-1} \mathfrak{h} \subset \mathfrak{h} \otimes_{\mathbb{R}} \mathbb{C}$ such that $\left[H_{0}, v\right]=v$ for any $v \in T_{0}(\Omega)$, so that, given any $H \in \mathfrak{h}$, replacing $H$ by $H^{b}:=H-\mu(H) H_{0}$ we obtain $H^{b} \in \mathfrak{h}$ such that $\mu\left(H^{b}\right)=0$. Finally, to verity $(\sharp)$ it remains to show that given any $\rho, \tau \in \Delta_{0}^{+}$, there exists $H \in \Delta_{0}^{+}$such that $\tau(H) \neq \rho(H)$ (hence also $\tau\left(H^{b}\right) \neq \rho\left(H^{\mathrm{b}}\right)$ ), which is obvious since $\Delta_{0}^{+}$spans $\sqrt{-1} \mathfrak{h}^{*}$ over $\mathbb{R}$. The proof of Lemma 1 is complete.

As will be seen later on, for practical purposes it is more convenient to do computations on a domain of type $A, D$ or $E$, in which case all the roots are of equal length. For that reason it is convenient to identify an irreducible bounded symmetric domain of type $B$ or of type $C$ as a complex submanifold of another irreducible bounded symmetric domain of type $A, D$ or $E$. More precisely, we have

Lemma 3. Let $\Omega^{\prime} \Subset \mathbb{C}^{N^{\prime}}$ be an irreducible bounded symmetric domain in its Harish-Chandra realization. Then, $\Omega_{0}$ can be embedded into an irreducible bounded symmetric domain $\Omega \Subset C^{N}$ of type $A, D$ or $E$ as a totally geodesic complex submanifold such that $\Omega_{0}$ is the intersection of $\Omega$ with a complex vector subspace of $\mathbb{C}^{N}$.

Proof. According to the classification given in $\S 1, \Omega$ is of type $A, D$ or $E$, except in the case of $D_{n}^{I I I}, n \geq 3$, which is of type $C_{n}$, and in the case of $D_{n}^{I V}$, when $n=2 k+1$ is odd, which is of type $B_{k+1}$. Clearly $D_{n}^{I I I} \subset D_{n, n}^{I}$, which is of type $A_{2 n-1}$ from the inclusion $M_{s}(n ; \mathbb{C}) \subset M(n, n ; \mathbb{C})$, where $M_{s}(n ; \mathbb{C})$ stands 
for the complex vector space of symmetric $n$-by- $n$ matrices with complex entries. Furthermore $D_{2 k+1}^{I V} \subset D_{2 k+2}^{I V}$, which is of type $D_{k+2}$, when $\left(z_{1}, \cdots, z_{n}\right)$ is identified with $\left(z_{1}, \cdots, z_{n}, 0\right)$. Clearly each of the embeddings $D_{n}^{I I I} \subset D_{n, n}^{I}$ and $D_{2 k+1}^{I V} \subset D_{2 k+2}^{I V}$ is given by the intersection of the ambient domains by complex vector subspace. Denote by $\left(\Omega^{\prime}, \Omega\right)$ a pair of bounded symmetric domains which is either $\left(D_{2 k+1}^{I V}, D_{2 k+2}^{I V}\right), k \geq 2$ or $\left(D_{n}^{I I I}, D_{n, n}^{I}\right), n \geq 3$, and by $\nu: \Omega^{\prime} \rightarrow \Omega$ the standard embedding. To prove Lemma 3 it remains to check that the embedding $\nu: \Omega^{\prime} \rightarrow \Omega$ is totally geodesic. To see this, denoting by $G_{0}^{\prime}$ the identity component of the automorphism group of $\Omega^{\prime}$ and by $K^{\prime} \subset G_{0}^{\prime}$ the isotropy subgroup at $0 \in \Omega^{\prime} \subset \Omega$, the holomorphic embedding $\nu: \Omega^{\prime} \cong G_{0}^{\prime} / K^{\prime} \rightarrow G_{0} / K \cong \Omega$ is equivariant with respect to a group homomorphism $\varphi: G_{0}^{\prime} \rightarrow G_{0}$, and to check total geodesy it suffices to check the second fundamental form at the point $0 \in \Omega^{\prime} \subset \Omega$. But since $\Omega$ is invariant under the symmetric $z \rightarrow-z$ at the origin, the Riemann-Christoffel symbols of $(\Omega, g)$ with respect to the canonical Kähler-Einstein metric $g$ vanish at 0 , and the second fundamental form of the linear section $\nu\left(\Omega^{\prime}\right)=\Omega \cap T_{0}\left(\Omega^{\prime}\right)$ is necessarily zero at $0 \in \Omega^{\prime} \subset \Omega$, proving that $\nu: \Omega^{\prime} \rightarrow \Omega$ is indeed totally geodesic. The proof of Lemma 3 is complete.

For the purpose of computing the canonical Kähler-Einstein metric in terms of Euclidean coordinates, it is convenient to make use of totally geodesic Hermitian symmetric manifolds of rank 1 , i.e., those isomorphic to $\mathbb{B}^{n}$. We have in particular the following lemma which explains why it is convenient to work with domains of type $A, D$ or $E$, where all roots are of equal length.

Lemma 4. Suppose $\Omega \Subset \mathbb{C}^{N}, \Omega=G_{0} / K$ in standard notation, is an irreducible bounded symmetric domain in its Harish-Chandra realization equipped with the canonical Kähler-Einstein metric $g$ such that minimal disks are of Gauss curvature -1. Suppose $\rho, \tau \in \Delta_{0}^{+}, \rho \neq \tau$, are of maximal length and $\rho-\tau$ is a root. Then, $D_{\rho, \tau}:=\left(\mathfrak{g}_{\rho} \oplus \mathfrak{g}_{\tau}\right) \cap \Omega$ is a totally geodesic complex submanifold isometrically biholomorphic to the complex unit 2-ball $\mathbb{B}^{2}$ equipped with the canonical Kähler-Einstein metric $h$ of constant holomorphic sectional curvature -1 .

Proof. We collect here some basic facts about maximal polydisks in the proof of the Polydisk Theorem stated in the above and refer the reader to Wolf [Wo]. Suppose the irreducible bounded symmetric domain $\Omega$ is of rank $r$. Let $\Pi \cong D^{r}$ be a standard maximal polydisk passing through $0 \in \Omega$ constructed from root 
spaces, as follows. A set of roots $\Phi \subset \Delta_{0}^{+}$is said to be strongly orthogonal if and only if $\rho \pm \tau \notin \Delta$ for any $\rho, \tau \in \Phi$. Let $\rho_{1}=\mu \in \Delta_{0}^{+}$be the highest root, and, for $k \geq 0$, let $\rho_{k+1}$ be inductively the highest root (with respect to some choice of a lexicographic ordering) in $\Delta_{0}^{+}$strongly orthogonal to $\left\{\rho_{1}, \cdots, \rho_{k}\right\}$, if the set of such roots is non-empty. Then, the maximal set $\Psi$ of strongly orthogonal roots constructed this way is of cardinality $r$, and the maximal polydisk $\Pi$ is the orbit of 0 under the group $G_{0}[\Psi]$ which is the direct product of $G_{0}[\rho], \rho \in \Psi$. As a consequence, $T_{0}(\Pi)$ is spanned by root spaces $\mathfrak{g}_{\rho}, \rho \in \Psi$. Moreover, all roots in $\Psi$ are of maximal length, so that $R\left(e_{\rho}, \overline{e_{\rho}} ; e_{\rho}, \overline{e_{\rho}}\right)=-1$ for any $\rho \in \Psi$. Recall that $\Omega=\bigcup\{\gamma \Pi: \gamma \in K\}$, so that any vector $\eta \in T_{0}(\Omega)$ is equivalent under the action of the isotropy group $K \subset G_{0}$ to a vector in $T_{0}(\Pi)$.

From the preceding discussion it follows that for any $\eta \in T_{0}(\Omega)$ of unit length with respect to $g$, we have $-1 \leq R_{\eta \bar{\eta}} \eta \bar{\eta} \leq-\frac{1}{r}, r=\operatorname{rank}(\Omega)$, and equality holds if and only if $\eta$ is a minimal rational tangent, in which case $\Omega \cap \mathbb{C} \eta$ is a minimal disk on $\Omega$. Let $e_{\rho} \in \mathfrak{g}_{\rho}, e_{\tau} \in \mathfrak{g}_{\tau}$ be unit vectors, and write $\xi=a e_{\rho}+b e_{\tau}$, $|a|^{2}+|b|^{2}=1$. Since $\rho$ and $\tau$ are positive noncompact roots of maximal length we have $R\left(e_{\rho}, \overline{e_{\rho}} ; e_{\rho}, \overline{e_{\rho}}\right)=R\left(e_{\tau}, \overline{e_{\tau}} ; e_{\tau}, \overline{e_{\tau}}\right)=-1$. In particular, $D_{\rho}:=\Omega \cap \mathbb{C} e_{\rho}$ and $D_{\tau}:=\Omega \cap \mathbb{C} e_{\tau}$ are minimal disks on $\Omega$. In the expansion for $R_{\xi \bar{\xi} \xi \bar{\xi}}$ we get (1)

$$
\begin{aligned}
R_{\xi \bar{\xi} \xi \bar{\xi}} & =|a|^{4} R\left(e_{\rho}, \overline{e_{\rho}} ; e_{\rho}, \overline{e_{\rho}}\right)+4|a|^{2}|b|^{2} R\left(e_{\rho}, \overline{e_{\rho}} ; e_{\tau}, \overline{e_{\tau}}\right)+|b|^{4} R\left(e_{\tau}, \overline{e_{\tau}} ; e_{\tau}, \overline{e_{\tau}}\right) \\
& =-\left(|a|^{4}+2|a|^{2}|b|^{2}+|b|^{4}\right)=-\left(|a|^{2}+|b|^{2}\right)^{2}=-1
\end{aligned}
$$

Here in the expansion for $R_{\xi \bar{\xi} \xi \bar{\xi}}$ we use the fact that $R\left(e_{\rho}, \overline{e_{\rho}} ; e_{\tau}, \overline{e_{\tau}}\right)=-\frac{1}{2}$ since $\rho-\tau$ is a root, and that all other terms in the expansion such as $R\left(e_{\rho}, \overline{e_{\rho}} ; e_{\rho}, \overline{e_{\tau}}\right)$ and $R\left(e_{\rho}, \overline{e_{\tau}} ; e_{\rho}, \overline{e_{\tau}}\right)$ are zero. To see the latter statement, taking $e_{-\rho}$ resp. $e_{-\tau}$ to be $\overline{e_{\rho}}$ resp. $\overline{e_{\tau}}$ and denoting by $B$ the (complex bilinear) Killing form on $\mathfrak{g}$, for some real number $c$ we have $R\left(e_{\rho}, \overline{e_{\rho}} ; e_{\rho}, \overline{e_{\tau}}\right)=c B\left(\left[e_{\rho}, \overline{e_{\rho}}\right],\left[e_{\rho}, \overline{e_{\tau}}\right]\right)=$ $c B\left(H_{\rho}, N_{\rho,-\tau} e_{\rho-\tau}\right)$, where $H_{\rho}:=\left[e_{\rho}, \overline{e_{-\rho}}\right]$ belongs to the Cartan subalgebra $\mathfrak{h} \subset \mathfrak{g}$ and $N_{\rho,-\tau}$ is some real constant, from which it follows readily that $R\left(e_{\rho}, \overline{e_{\rho}} ; e_{\rho}, \overline{e_{\tau}}\right)=0$. Similarly, we have $R\left(e_{\rho}, \overline{e_{\tau}} ; e_{\rho}, \overline{e_{\tau}}\right)=c B\left(\left[e_{\rho}, \overline{e_{\tau}}\right], \overline{\left[e_{\tau}, \overline{e_{\rho}}\right]}\right)=$ $c B\left(N_{\rho,-\tau} e_{\rho-\tau}, \overline{N_{\tau,-\rho} e_{\tau-\rho}}\right)=0$, since the root spaces are mutually orthogonal on $\mathfrak{g}$ with respect to the Hermitian bilinear form $B\left(\cdot,,^{-}\right)$induced by the Killing form $B$. (Alternatively, one can check the curvature identities $R\left(e_{\rho}, \overline{e_{\rho}} ; e_{\rho}, \overline{e_{\tau}}\right)=$ $R\left(e_{\rho}, \overline{e_{\tau}} ; e_{\rho}, \overline{e_{\tau}}\right)=0$ from curvature inequalities on Hermitian symmetric spaces 
as in Mok-Zhong [MZ]). As to the former statement that $R\left(e_{\rho}, \overline{e_{\rho}} ; e_{\tau}, \overline{e_{\tau}}\right)=-\frac{1}{2}$ we have $R\left(e_{\rho}, \overline{e_{\rho}} ; e_{\rho}, \overline{e_{\rho}}\right)=c B\left(H_{\rho}, \overline{H_{\rho}}\right)$ while

$$
\begin{aligned}
& R\left(e_{\rho}, \overline{e_{\rho}} ; e_{\tau}, \overline{e_{\tau}}\right)=R\left(e_{\rho}, \overline{e_{\tau}} ; e_{\tau}, \overline{e_{\rho}}\right)=c B\left(\left[e_{\rho}, \overline{e_{\tau}}\right], \overline{\left[e_{\rho}, \overline{e_{\tau}}\right]}\right) \\
& =c B\left(N_{\rho,-\tau} e_{\rho-\tau}, \overline{N_{\rho,-\tau} e_{\rho-\tau}}\right)=c N_{\rho,-\tau}^{2} B\left(e_{\rho-\tau}, \overline{e_{\rho-\tau}}\right) .
\end{aligned}
$$

On the other hand, since the maximal $(-\tau)$-chain associated to the root $\rho \in \Delta$ is given by $(\rho, \rho-\tau)$, we have $N_{\rho,-\tau}^{2}=\frac{1}{2} B\left(H_{\rho-\tau}, \overline{H_{\rho-\tau}}\right)$ (cf. Helgason [Hel], p.176). Since all roots are of equal length, it follows by comparing curvature formulas above that $R\left(e_{\rho}, \overline{e_{\rho}} ; e_{\tau}, \overline{e_{\tau}}\right)=-\frac{1}{2} R\left(e_{\rho}, \overline{e_{\rho}} ; e_{\rho}, \overline{e_{\rho}}\right)=-\frac{1}{2}$, completing the proof of the curvature identity (1).

From (1) it follows that any $\xi \in \mathfrak{g}_{\rho} \oplus \mathfrak{g}_{\tau}$ is a minimal rational tangent. Since $D_{\rho, \tau}=\left(\mathfrak{g}_{\rho} \oplus \mathfrak{g}_{\tau}\right) \cap \Omega \subset \Omega$ is the intersection of $\Omega$ with a vector subspace, for any $x \in D_{\rho, \tau}$, the tangent space $T_{x}\left(D_{\rho, \tau}\right)$ is identified with $T_{0}\left(D_{\rho, \tau}\right)$ in the trivialization $T(\Omega)=T_{0}(\Omega) \times \Omega$. Hence, by Lemma 1 , for any $x \in D_{\rho, \tau}$, any $\xi(x) \in T_{x}\left(D_{\rho, \tau}\right)$ is a minimal rational tangent. Let $\sigma$ be the second fundamental form of $D_{\rho, \tau}$ in $\Omega$ with respect to the canonical Kähler-Einstein metric $g$ on $\Omega$. Since any $\xi(x) \in T_{x}\left(D_{\rho, \tau}\right)$ is tangent to a minimal disk lying on $D_{\rho, \tau}$ it follows that $\sigma(\xi(x), \xi(x))=0$ for any $\xi(x) \in T_{x}\left(D_{\rho, \tau}\right)$. By polarization we conclude that $\sigma \equiv 0$ on $D_{\rho, \tau}$. As a consequence $D_{\rho, \tau} \subset \Omega$ is a totally geodesic complex submanifold. Since $\left(D_{\rho, \tau} ;\left.g\right|_{D_{\rho, \tau}}\right)$ is of constant holomorphic sectional curvature -1 it must be of rank 1 and hence isometrically biholomorphic to the complex unit 2-ball $\mathbb{B}^{2}$ equipped with the canonical Kähler-Einstein metric $h$ of constant holomorphic sectional curvature -1 . The proof of Lemma 4 is complete.

Using Lemma 4 and adopting the notation of Lemma 2, we have the following explicit description of the canonical Kähler-Einstein metric along a minimal disk.

Lemma 5. Let $\Omega \Subset \mathbb{C}^{N}$ be an irreducible bounded symmetric domain of type $A$, $D$ or $E$ in its Harish-Chandra realization, $\Omega=G_{0} / K$, equipped with the canonical Kähler-Einstein metric such that minimal disks on $\Omega$ are of Gauss curvature -1 . Fix a Cartan subalgebra $\mathfrak{h} \subset \mathfrak{k}$ and let $\left\{e_{k}\right\}_{k=1}^{N}$ be a basis of $T_{0}(\Omega) \cong \mathbb{C}^{N}$ consisting of root vectors of unit length, with $e_{1}$ belonging to the highest root vector $\mu \in \Delta_{0}^{+}$, $e_{k}$ belonging to some root $\rho \in \mathfrak{H}$ for $2 \leq k \leq p+1$, and $e_{\ell}$ belonging to some root $\tau \in \mathfrak{N}$ for $p+2 \leq \ell \leq N$. Write $D_{0}:=\mathbb{C}_{1} \cap \Omega$, which is a minimal disk. Then, 
for $\left(z_{1}, 0, \cdots, 0\right) \in D_{0}$ we have

$$
\begin{gathered}
g_{1 \overline{1}}=\frac{2}{\left(1-\left|z_{1}\right|^{2}\right)^{2}} ; \quad g_{k \bar{k}}=\frac{2}{1-\left|z_{1}\right|^{2}} \text { for } 2 \leq k \leq p+1 ; \\
g_{\ell \bar{\ell}}=2 \text { for } p+2 \leq \ell \leq N ; \quad g_{i \bar{j}}=0 \text { for } i \neq j, 1 \leq i, j \leq N .
\end{gathered}
$$

Proof. Since $e_{1}$ belongs to a highest root $\mu$, it is a minimal rational tangent, so that $D_{0}=\mathbb{C} \cap \Omega$ is a minimal disk on $(\Omega, g)$. By Lemma 2 the metric tensor $g$ is diagonalized along the minimal disk $D_{0}$ when one uses a basis consisting of root vectors of unit length. Thus,

$$
g_{i \bar{j}}=0 \text { for } i \neq j, 1 \leq i, j \leq N
$$

Since all roots are of the same length for $\Omega$ of type $A, D$ or $E$ and since $\mu-\tau \in \Delta_{0}^{+}$ by assumption, by Lemma $2 D_{\mu, \tau}:=\left(\mathfrak{g}_{\mu}+\mathfrak{g}_{\tau}\right) \cap \Omega \subset \Omega$ is a totally geodesic complex submanifold such that $\left(D_{\mu, \tau} ;\left.g\right|_{D_{\mu, \tau}}\right)$ is a totally geodesic submanifold of $(\Omega, g)$ isomorphic to the complex unit 2-ball $\left(\mathbb{B}^{2}, h\right)$ equipped with the canonical Kähler-Einstein metric of constant holomorphic sectional curvature -1 . As is well-known, on $\left(\mathbb{B}^{2}, h\right)$ the metric tensor $\left(h_{i \bar{j}}\right)$ along the minimal disk $\mathbb{B}^{1} \subset \mathbb{B}^{2}$, $\mathbb{B}^{1} \times\{0\}$, is given by

$$
h_{1 \overline{1}}=\frac{2}{\left(1-\left|z_{1}\right|^{2}\right)^{2}} ; \quad h_{2 \overline{2}}=\frac{2}{1-\left|z_{1}\right|^{2}} ; \quad h_{1 \overline{2}}=h_{2 \overline{1}}=0 .
$$

On the other hand, if $\mu-\tau \notin \Delta_{0}^{+}$, then $\mu$ and $\tau$ are strongly orthogonal to each other, and $D_{\mu, \tau}:=\left(\mathfrak{g}_{\mu}+\mathfrak{g}_{\tau}\right) \cap \Omega$ is again a totally geodesic complex submanifold isomorphic to the bidisk $\left(D^{2}, s\right)=\left(D, d s_{D}^{2}\right) \times\left(D, d s_{D}^{2}\right)$, where $d s_{D}^{2}$ denotes the Poincaré metric of constant Gaussian curvature -1 on the unit disk $D$. On $\left(D^{2}, s\right)$ we have

$$
s_{1 \overline{1}}=\frac{2}{\left(1-\left|z_{1}\right|^{2}\right)^{2}} ; \quad s_{2 \overline{2}}=2 ; \quad s_{1 \overline{2}}=s_{2 \overline{1}}=0
$$

The proof of Lemma 5 is complete.

$\S 3$ Asymptotic behavior of the second fundamental form along a holomorphic curve exiting along smooth points of $\partial \Omega$ 
(3.1) For the study of holomorphic curves on a bounded symmetric domain $\Omega$ in its Harish-Chandra realization exiting along $\operatorname{Reg}(\partial \Omega)$, the smooth part of an irreducible bounded symmetric domain $\Omega$, we will first prove a transversality statement. For its proof we will need to make use of some bounded exhaustion function on $\Omega$ which extends to a smooth function on a neighborhood of $\bar{\Omega}$ arising from Herman [Her] in the description of $\Omega$ as the unit ball with respect to some Banach norm.

Lemma 6. Let $\Omega \Subset \mathbb{C}^{N}$ be a bounded symmetric domain in its Harish-Chandra realization. Then, there exists a convex bounded exhaustion function $\rho$ defined on a neighborhood of $\bar{\Omega}$ such that for any smooth boundary point $b \in \partial \Omega, \rho$ is defined and smooth on some neighborhood $U_{b}$ of $b$ in $\mathbb{C}^{N}$.

Proof. By the Herman Convexity Theorem (Herman [Her], cf. Wolf [Wo]), $\Omega \Subset \mathbb{C}^{N}$ can be identified as the unit ball in $T_{0}(\Omega) \cong \mathbb{C}^{N}$ with respect to the Hermann norm $\|\cdot\|_{H}$. Define $\rho(z)=\|z\|_{H}-1$ for $z \in \mathbb{C}^{N}$. Then, $\rho$ is in fact defined everywhere on $\mathbb{C}^{N}$, and its restriction to $\Omega$ is a bounded convex exhaustion function on $\Omega$. In particular, $\rho$ is a bounded plurisubharmonic exhaustion function on $\Omega$. Now, the norm $\|z\|_{H}$ is smooth on a neighborhood of $z \in \mathbb{C}^{N}$ if and only if the level sets of $\rho$ are smooth real hypersurfaces on a neighborhood of $z$, which holds true for $z=b \in \partial \Omega$ a smooth point of $\partial \Omega$.

We are ready to formulate and prove the transversality statement.

Lemma 7. For the unit disk $D$ let $b_{0} \in \partial D, U$ be an open neighborhood of $b_{0}$ of the form $U=D\left(b_{0} ; \epsilon\right)$ for some $\epsilon>0, f: U \rightarrow \mathbb{C}^{N}$ be a holomorphic embedding such that $f(U \cap D) \subset \Omega, f(U \cap \partial D) \subset \partial \Omega$. Suppose for any $b \in U \cap \partial D, f(b)$ is actually a smooth point of $\partial \Omega$. Then, for a general point $b \in U \cap \partial D, \partial f(b)$ is transversal to the complex tangent space $T_{f(b)}(\partial \Omega) \cong \mathbb{C}^{n-1}$.

Proof. We argue by contradiction. Consider the function $\varphi(w)=\rho(f(w))$ defined on $U$. We have $\varphi(w)<0$ for $w \in U \cap D$ and $\varphi(w)=0$ for $z \in U \cap \partial D$. Suppose $d f(w) \in T_{f(w)}^{1,0}(\partial \Omega)$ for any $w \in U \cap \partial D$. For a general point $b \in U \cap \partial D$, i.e., for all $b$ on the circular arc $U \cap \partial D$ except for a discrete subset of the latter, there exists $\epsilon_{b}>0$ such that, writing $U_{b}=D\left(b ; \epsilon_{b}\right)$, the smooth function $\varphi$ vanishes along $U_{b} \cap \partial D$ exactly to the order $k \geq 1$ (where a priori the integer $k$ depends on $b)$. 
Write $\psi=|w|^{2}-1<0$ on $D$. Then on $U_{b}$ we have $-\varphi=(-\psi)^{k} h$ where $h$ is smooth and positive on $U_{b}$, i.e., $\varphi=(-1)^{k-1} \psi^{k} h$. We have

$$
\begin{aligned}
0 \leq \sqrt{-1} \partial \bar{\partial} \varphi= & (-1)^{k-1} h \sqrt{-1} \partial \bar{\partial}\left(\psi^{k}\right)+(-1)^{k-1} \psi^{k} \sqrt{-1} \partial \bar{\partial} h \\
& +(-1)^{k-1} \sqrt{-1} \partial\left(\psi^{k}\right) \wedge \bar{\partial} h+(-1)^{k-1} \sqrt{-1} \partial h \wedge \bar{\partial}\left(\psi^{k}\right)
\end{aligned}
$$

Noting that

$\sqrt{-1} \partial \bar{\partial}\left(\psi^{k}\right)=\sqrt{-1} \partial\left(k \psi^{k-1} \bar{\partial} \psi\right)=k(k-1) \psi^{k-2} \sqrt{-1} \partial \psi \wedge \bar{\partial} \psi+k \psi^{k-1}(\sqrt{-1} \partial \bar{\partial} \psi)$

$$
=k(k-1) \psi^{k-2} \sqrt{-1} \partial \psi \wedge \bar{\partial} \psi+O\left(|\psi|^{k-1}\right),
$$

substituting (2) into (1) we have

$$
\begin{gathered}
0 \leq \sqrt{-1} \partial \bar{\partial} \varphi=(-1)^{k-1} k(k-1) h \psi^{k-2} \sqrt{-1} \partial \psi \wedge \bar{\partial} \psi+O\left(|\psi|^{k-1}\right) \\
=-k(k-1) h(-\psi)^{k-2} \sqrt{-1} \partial \psi \wedge \bar{\partial} \psi+O\left(|\psi|^{k-1}\right)
\end{gathered}
$$

Since $|d \psi| \neq 0$ on a neighborhood of the unit circle the right hand side is strictly negative at $w \in U \cap D$ sufficiently close to $b$ and, we have reached a contradiction unless $k=1$, as desired.

For the proof of the Main Theorem we will need the following lemma which describes the complex tangent space at a smooth boundary point of an irreducible bounded symmetric domain $\Omega \Subset \mathbb{C}^{N}$. In what follows for a smooth point $y \in \partial \Omega$, $T_{y}^{1,0}(\partial \Omega) \subset T_{y}\left(\mathbb{C}^{N}\right)$ stands for the complex vector subspace of $(1,0)$-vectors tangent to $\partial \Omega$, where the holomorphic tangent space $T_{y}\left(\mathbb{C}^{N}\right)$ is canonically identified with the complex vector space $T_{y}^{1,0}\left(\mathbb{C}^{N}\right)$ of complexified tangent vectors of type $(1,0)$.

Lemma 8. In the notation of Lemma 7, for a general point $b \in U \cap \partial D$, we have $f^{*} g(w)=\left(\frac{2}{\left(1-|w|^{2}\right)^{2}}+s(w)\right)|d w|^{2}$ on $U_{b} \cap D$, where $U_{b}=D\left(b ; \epsilon_{b}\right) \subset U$ for some $\epsilon_{b}>0$, and $s$ is a smooth function defined on a neighborhood of $\overline{U_{b}}$.

Proof. We start by describing a potential function for the canonical KählerEinstein metric $g$ on the irreducible bounded symmetric domain $\Omega$, recalling that $g$ is normalized so that minimal disks on $\Omega$ are of Gauss curvature -1 . Consider the Bergman metric $d s_{\Omega}^{2}$ on $\Omega$, noting that $g=A d s_{\Omega}^{2}$ for some constant $A>0$. 
Denote by $\omega_{\Omega}$ the Kähler form of $\left(\Omega, d s_{\Omega}^{2}\right)$, and by $K_{\Omega}\left(z, z^{\prime}\right)$ the Bergman kernel on $\Omega$, which can be described as follows (cf. Faraut-Korányi [FK, pp.76-77]). We have $K_{\Omega}\left(z, z^{\prime}\right)=\frac{C_{\Omega}}{h(z, \zeta)^{m}}$, where $C_{\Omega}>0, m$ is a positive integer and $h$ is a polynomial in $\left(z_{1}, \cdots, z_{N} ; \overline{z_{1}^{\prime}}, \cdots, \overline{z_{N}^{\prime}}\right)$ with the following property (cf. FarautKorányi $\left[\mathrm{FK}\right.$, pp.76-77]). Let $\Pi \cong D^{r}$ be a maximal polydisk on $\Omega$ passing through 0 . We may choose Harish-Chandra coordinates such that $\Pi$ is exactly the unit polydisk $D^{r} \times\{0\}$. For $z \in \Omega$, there exists $\gamma \in K=\operatorname{Aut}_{0}(\Omega)$ such that $\gamma(z)=\left(a_{1}, \cdots, a_{r}\right) \in \Pi$ and we have

$$
h(z, z)=\left(1-\left|a_{1}\right|^{2}\right) \times \cdots \times\left(1-\left|a_{r}\right|^{2}\right) .
$$

The function $\tau(z)=-h(z, z)$ is in fact a defining function for $U \cap \Omega$, vanishing exactly to the order 1 along $U \cap \partial D$. (As an example, we have $\tau(z)=\operatorname{det}\left(I-\bar{Z}^{t} Z\right.$ ) in the case of a type- 1 domain $\Omega=D_{p, q}^{I}$.) Then, $\omega_{\Omega}(z)=\sqrt{-1} \partial \bar{\partial} \log K(z, z)=$ $-m \sqrt{-1} \partial \bar{\partial} \log h(z, z)$. Restricting $d s_{\Omega}^{2}$ to a minimal disk of $\Omega$, e.g., to any of the coordinate disks of $\Pi$, we see that minimal disks are of Gauss curvature $-\frac{2}{m}$ on $\Omega$. By the normalization on $g$ that minimal disks are of Gauss curvature -1 it follows that the Kähler form $\omega_{g}$ is given by $\omega_{g}=-\frac{m}{2} \omega_{\Omega}^{2}=-2 \sqrt{-1} \partial \bar{\partial} \log h(z, z)$. Thus $-2 \log h(z, z)=-2 \log (-\tau)$ is a potential function for $g$ on $\Omega$.

The Poincaré metric $d s_{D}^{2}$ on $D$ is given by $d s_{D}^{2}=\frac{2|d w|^{2}}{\left(1-|w|^{2}\right)^{2}}$, and the associated Kähler form $\omega_{D}$ is given by $\omega_{D}=\sqrt{-1} \partial \bar{\partial}\left(-2 \log \left(1-|w|^{2}\right)\right)$. Write $\varphi(w)=-2 \log \left(1-|w|^{2}\right)$. We have $f^{*} \omega_{g}=-2 \sqrt{-1} \partial \bar{\partial} \log (-\tau(f(w)))$. From the transversality result given in Lemma 7 , choosing $b \in \partial D$ generic and $\epsilon_{b}>0$ sufficiently small, $-\tau(f(w))$ vanishes exactly to the order 1 on a neighborhood of $U_{b} \cap D$ on $\partial D$ (where $U_{b}=D\left(b, \epsilon_{b}\right)$ ), and $\tau(f(w))=\left(1-|w|^{2}\right) \chi(w)$ where $\chi$ is a smooth positive function defined on some neighborhood of $\overline{U_{b}}$. It follows that $f^{*} \omega_{g}=\sqrt{-1} \partial \bar{\partial} \varphi+\sqrt{-1} \partial \bar{\partial} \log \chi=\left(\frac{2}{\left(1-|w|^{2}\right)^{2}}+s(w)\right)(\sqrt{-1} d w \wedge d \bar{w})$, where $s=\frac{\partial^{2} \log \chi}{\partial w \partial \bar{w}}$, and Lemma 8 follows.

We proceed to describe complex tangent spaces $T_{y}^{1,0}(\partial \Omega)$ for $y \in \operatorname{Reg}(\partial \Omega)$.

Lemma 9. Let $\Omega \Subset \mathbb{C}^{N}$ be an irreducible bounded symmetric domain in its Harish-Chandra realization. Let $\alpha \in T_{0}(\Omega)$ be a minimal rational tangent at the origin $0 \in \mathbb{C}^{N}$ and write $D_{\alpha}$ for the minimal disk passing through 0 and tangent to $\alpha$. Suppose $y \in \partial D_{\alpha}$. Then, $y \in \partial \Omega$ is a smooth boundary point and the 
complex tangent space $T_{y}^{1,0}(\partial \Omega) \subset T_{y}\left(\mathbb{C}^{N}\right)$ is the orthogonal complement of $\mathbb{C} \alpha$ a with respect to the Euclidean metric. (Here $T_{y}\left(\mathbb{C}^{N}\right)$ is identified with $\mathbb{C}^{N}$ in terms of the standard trivialization of $T\left(\mathbb{C}^{N}\right)$ given by the Euclidean coordinates, and $\mathbb{C} \alpha \subset \mathbb{C}^{N}$ is regarded thus as a vector subspace of $T_{y}\left(\mathbb{C}^{N}\right)$.)

Proof. We will use notations as those in Lemma 2. Let $\mu \in \Delta_{0}^{+}$be the highest root. Modulo the action of the isotropy subgroup it suffices to consider the case where $\alpha \in \mathfrak{g}_{\mu}$. Denote by $K_{\alpha} \subset K$ the subgroup consisting of $\gamma \in K$ such that $d \gamma(\alpha)=\alpha$. Since $K$ acts on $\mathbb{C}^{N}$ as linear transformations so that $\gamma(z)=d \gamma(0)(z)$ for any $z \in \mathbb{C}^{N}$, where we identify $T_{0}\left(\mathbb{C}^{N}\right)$ with $\mathbb{C}^{N}$ canonically, we must have $\gamma(y)=y$ for any $y \in K_{\alpha}$. As a consequence, the complex tangent space $T_{y}^{1,0}(\partial \Omega) \subset T_{y}\left(\mathbb{C}^{N}\right)$ is a complex hyperplane invariant under $K_{\alpha}$. Let now $V \subset T_{y}\left(\mathbb{C}^{N}\right)$ be the orthogonal complement of $\alpha$ (which is identified as a tangent vector at $y$ by Euclidean translation). To prove Lemma 8 it remains to show that $V=T_{y}^{1,0}(\partial \Omega)$. Suppose otherwise. Then, $W:=V \cap T_{y}^{1,0}(\partial \Omega) \subset T_{y}\left(\mathbb{C}^{N}\right)$ is of codimension 1 in $T_{y}^{1,0}(\partial \Omega)$, and the orthogonal complement $E$ of $W$ in $V$ is given by $\mathbb{C} \eta$, where $\eta=\alpha+\beta$ for some non-zero $\beta \in V$, and $E=\mathbb{C} \eta$ is invariant under $K_{\alpha}$. Expressing $\beta=\sum_{\rho \in \Theta} b_{\rho} e_{\rho}, b_{\rho} \neq 0$, where the summation is performed over a non-empty set of $\Theta \subset \Delta_{0}^{+}-\{\mu\}$. Fixing $\rho \in \Theta$, by the proof of Lemma 2 there exists some $H \in \mathfrak{h}$ such that $\alpha(H)=0$ and $\rho(H) \neq 0$. Then, $\exp (\mathbb{R} H) \subset K_{\alpha}$ and $e^{t H}(\eta), \eta=\alpha+\beta$, is of the form $\alpha+\beta^{\prime}(t)$, where $\beta^{\prime}(t) \neq \beta$ for $t \in \mathbb{R}$ nonzero and sufficiently small. Hence, $E$ is not invariant under $\exp (\mathbb{R} H)$, a plain contradiction. Thus, $V=T_{y}^{1,0}(\partial \Omega)$ and the proof of Lemma 9 is complete.

(3.2) Reduction of the proof of Main Theorem in the irreducible case We are now ready to give a proof of Main Theorem. Since the proof is long it will be given in three separate subsections, starting in this subsection with a reduction of the proof. Only one system of numbering of equations will be used throughout the proof of Main Theorem. We have the following reduction of the proof of Main Theorem in the case where $\Omega$ is an irreducible bounded symmetric domain.

Proposition 1. Let $\Omega \Subset \mathbb{C}^{N}$ be an irreducible bounded symmetric domain. Let $g$ be the canonical Kähler-Einstein metric on $\Omega$ normalized so that minimal disks are of constant Gauss curvature -1. In the notation of Main Theorem, for $w \in U \cap D$ denote by $\eta(w) \in T_{f(w)}(\Omega)$ a $(1,0)$-vector of unit length with respect 
to $g$. Then, to prove Main Theorem it suffices to prove that, as $w$ approaches a general point $b \in U \cap \partial D$

$$
\text { (†) } \quad R_{\eta(w) \overline{\eta(w)} \eta(w) \overline{\eta(w)}}=-1+O\left(\delta(w)^{2}\right)
$$

Furthermore, $(\dagger)$ is satisfied provided that we have a decomposition $\eta(w)=$ $\alpha(w)+\zeta(w)$, where $\alpha$ is a minimal rational tangent at $f(w) \in \Omega$, and $\zeta(w) \in$ $T_{f(w)}(\Omega)$ satisfies $R\left(\alpha(w), \overline{\zeta(w)} ; \nu, \overline{\nu^{\prime}}\right)=0$, such that $\|\zeta(w)\|=O(\delta(w))$.

Proof of Proposition 1. Write $r=\operatorname{rank}(\Omega)$. For any unit tangent vector $\eta$ of type $(1,0)$ on $(\Omega, g)$, we have

$$
-1 \leq R_{\eta \bar{\eta} \eta \bar{\eta}} \leq-\frac{1}{r}
$$

and equality holds if and only if $\eta$ is a minimal rational tangent. Consider now the Hermitian Riemann surface $\left(U \cap D, f^{*} g\right)$. On the one hand, for $w \in U \cap \partial D$, writing $\eta=\eta(w)$ for a unit tangent vector proportional to $\left.d f\left(\frac{\partial}{\partial w}\right)\right)(w)$, we have (2) Gauss curvature $\left(U \cap D, f^{*} g\right)=R_{\eta(w) \overline{\eta(w)} \eta(w) \overline{\eta(w)}}-\|\sigma(f(w))\|^{2}$.

On the other hand, as $w \in U \cap D$ approaches a general point, by Lemma 8, $f^{*} g=\left(\frac{2}{\left(1-|w|^{2}\right)^{2}}+s(w)\right)|d w|^{2}$ for $w \in U \cap D$ near $b$, where $s(w)$ is a smooth function defined on some neighborhood of $\overline{U_{b}}$, where $U_{b}=D\left(b ; \epsilon_{b}\right) \Subset U$ for some $\epsilon_{b}>0$. Thus, writing $d s_{D}^{2}=\frac{2}{\left(1-|w|^{2}\right)^{2}}$ for the Poincaré metric on $D$ of constant Gauss curvature -1 , we have $f^{*} g=u d s_{D}^{2}$, where $u$ is a smooth function defined on a neighborhood of $\overline{U_{b}}$, and $h(w)=1+O\left(\delta(w)^{2}\right)$ on $U_{b}$. Write $f^{*} g=h|d w|^{2}$, so that $h=\frac{2 u}{\left(1-|w|^{2}\right)^{2}}$. Noting the curvature formula

(3) (Gauss curvature $\left.\left(U \cap D, f^{*} g\right)\right) \cdot \sqrt{-1} d w \wedge d \bar{w}=-\sqrt{-1} \partial \bar{\partial} \log h$, we have on $U_{b}$

(4) Gauss curvature $\left(U \cap D, f^{*} g\right) \cdot\left\|\frac{\partial}{\partial w}\right\|_{f^{*} g}^{2}=\frac{-2}{\left(1-|w|^{2}\right)^{2}}+O(1)$,

Noting that $\left\|\frac{\partial}{\partial w}\right\|_{f^{*} g}^{2}=\frac{2 u}{\left(1-|w|^{2}\right)^{2}}=\frac{2\left(1+O\left(\delta(w)^{2}\right)\right)}{\left(1-|w|^{2}\right)^{2}}$, and writing $\log h=-2 \log (1-$ $\left.|w|^{2}\right)+\log (2 u)$, we conclude that

$$
\text { Gauss curvature }\left(U \cap D, f^{*} g\right)=-1+O\left(\delta(w)^{2}\right)
$$


on $U_{b}$. Combining (1), (2) and (5), to prove Main Theorem it suffices to show that

$$
R_{\eta(w) \overline{\eta(w)} \eta(w) \overline{\eta(w)}}=-1+O(\delta(w))^{2} .
$$

We proceed to give a reformulation of $(\dagger)$. Let $\Pi \subset \Omega$ be a maximal polydisk, $\Pi \cong D^{r}$, passing through $0 \in \Omega$. Any vector $\eta \in T(\Omega)$ is equivalent under the $G_{0}=\operatorname{Aut}_{0}(\Omega)$ to some $\xi \in T_{0}(\Pi) \subset T_{0}(\Omega)$ (cf. the Polydisk Theorem), which can be chosen to be $\xi=\left(\xi_{1}, \cdots, \xi_{r}\right)$ in terms of the Euclidean coordinates on $\Pi \cong D^{r}$, where $\xi_{k}(w)$ is real, $1 \leq k \leq r$, and $\xi_{1} \geq \cdots \geq \xi_{r} \geq 0$. We call $\xi=\left(\xi_{1}, \cdots, \xi_{r}\right)$ the normal form of $\eta$ under $G_{0}$. For $w \in U \cap D$ we write $\eta_{w}=\left(\xi_{1}(w), \cdots, \xi_{r}(w)\right) \in T_{0}(\Pi)$ for the normal form of $\eta(w) \in T_{f(w)}(\Omega)$ under $G_{0}$. Thus, writing $\sqrt{2} \alpha=(1,0, \ldots, 0)$ we have $\eta_{w}=\sqrt{1-(c(w))^{2}} \alpha+\zeta_{w}$, where $\sqrt{2} \zeta_{w}=\left(0, \xi_{2}(w), \ldots, \xi_{r}(w)\right)$, and $c(w):=\left\|\zeta_{w}\right\|=\sqrt{\left|\xi_{2}\right|^{2}+\cdots+\left|\xi_{r}\right|^{2}}$, where $\|\cdot\|$ denotes $\|\cdot\|_{g}$. Clearly $R\left(\alpha, \overline{\zeta_{w}} ; \nu, \overline{\nu^{\prime}}\right)=0$ for any $\nu, \nu^{\prime} \in T_{0}(\Omega)$. As a consequence we have

$$
R_{\eta_{w} \overline{\eta_{w}} \eta_{w} \overline{\eta_{w}}}=\left(1-(c(w))^{2}\right)^{2} R_{\alpha \bar{\alpha} \alpha \bar{\alpha}}+R_{\zeta_{w} \overline{\zeta_{w}} \zeta_{w} \overline{\zeta_{w}}}=-1+2\left\|\zeta_{w}\right\|^{2}+O\left(\left\|\zeta_{w}\right\|^{4}\right) .
$$

Thus, to prove $(\dagger)$ it is sufficient to show

$$
(\dagger)^{\prime} \quad\left\|\zeta_{w}\right\|=O(\delta(w)) \text { on } U_{b} \cap D,
$$

which is equivalent to the last statement $\|\zeta(w)\|=O(\delta(w))$ in the statement of Proposition 1.

Since by assumption $f(b) \in \operatorname{Reg}(\partial \Omega)$, regarding $\Omega$ canonically as an open subset of $T_{0}(\Omega)$ the normal form of $f(b)$ under the action on $T_{0}(\Omega)$ of the isotropy group $K \subset G_{0}$ at 0 is given by $\left(1, a_{2}, \ldots, a_{r}\right) \in \operatorname{Reg}(\partial \Pi)$, where $1>a_{2} \geq \cdots \geq a_{r} \geq 0$. In other words, there exists $\gamma \in K$ such that $\gamma(f(b))=\left(1, a_{2}, \ldots, a_{r}\right) \in \operatorname{Reg}(\partial \Pi)$. There exists an automorphism $\chi_{0}$ in $\operatorname{Aut}_{0}(\Pi)$ such that $\chi_{0}\left(1, a_{2}, \ldots, a_{r}\right)=(1,0, \ldots, 0)$. The automorphism $\chi_{0}$ of $\Pi$ extends to an automorphism $\chi$ of $\Omega$, and $\chi \circ \gamma$ gives an automorphism $\chi$ of $\Omega$ such that $(\chi \circ \gamma)(f(b))=\chi(\gamma(f(b))=(1,0, \ldots, 0)$. For the proof of Main Theorem, without loss of generality we may assume that $f(b)=(1,0, \ldots, 0) \in \operatorname{Reg}(\partial \Pi)$.

Recall that a maximal polydisk $\Pi \subset \Omega$ can be constructed as follows. Writing $\Omega=G_{0} / K$ as before and fixing a Cartan subalgebra $\mathfrak{h} \subset \mathfrak{k}$, we have a maximal set 
$\Psi$ of strongly orthogonal positive noncompact roots $\psi_{i} \in \Psi, 1 \leq i \leq r$, and $\Pi \subset \Omega$ is the totally geodesic complex submanifold passing through $0=e K$ such that $T_{0}(\Pi)=\mathfrak{g}_{\psi_{1}} \oplus \cdots \oplus \mathfrak{g}_{\psi_{r}}$, where for a root $\rho$ with respect to the Cartan subalgebra $\mathfrak{h}, \mathfrak{g}_{\rho} \subset \mathfrak{g}^{\mathbb{C}}$ denotes the (complex 1-dimensional) root subspace belonging to $\rho$. In what follows we choose Euclidean coordinates $\left(z_{1}, \cdots, z_{N}\right)$ on $\mathbb{C}^{N}$, such that a point $\left(z_{1}, \cdots, z_{r}\right) \in \Pi$ corresponds to $\left(z_{1}, \cdots, z_{r}, 0, \cdots, 0\right) \in \mathbb{C}^{N}$, and such that, writing $e_{i}:=\frac{\partial}{\partial z_{i}}$ for $1 \leq i \leq n$. each $e_{i}$ is a root vector belonging to some noncompact positive root $\rho$ with respect to the Cartan subalgebra $\mathfrak{h}$. Write $y=(1,0, \cdots, 0)$. Denote by $\rho$ the defining function of $\Omega$ given by $\rho(z)=\|z\|_{H}-1$ as in Lemma 7 which is smooth on a neighborhood of $y$. Choosing $b \in U \cap \partial D$ to be a general point, by Lemma 7 we know that

$$
d \rho(y)\left(d f\left(\frac{\partial}{\partial w}\right)\right) \neq 0 .
$$

By Lemma $9, T^{1,0}(\partial \Omega)$ is the orthogonal complement of $e_{1}$ in $T_{y}(\partial \Omega) \cong \mathbb{C}^{N}$. It follows hence from $(7)$ that, writing $f(z)=\left(f_{1}(z), \cdots, f_{n}(z)\right)$, we have

$$
f_{1}^{\prime}(b) \neq 0 \text {. }
$$

We are going to deduce $(\dagger)$ from $(8)$. To illustrate the idea we will consider first of all the very special case where $\Omega$ is a polydisk.

(3.3) Proof of Main Theorem of the special case of polydisks In the notation of Main Theorem we assume now that $\Omega=\Pi=D^{r}$ is a polydisk. In the statement of Proposition 1 in (3.2), we assume that $\Omega$ is irreducible. The assumption was made so that we have only one type of minimal disks, which are all equivalent to one another under $G_{0}=\operatorname{Aut}_{0}(\Omega)$, and the canonical Kähler-Einstein metric can be normalized so that all minimal disks are of constant Gauss curvature -1 . In the general case, there is a type of minimal disk arising from each of the irreducible factors, and a normalization of the canonical Kähler-Einstein metric so that all types of minimal disks are of constant Gauss curvature -1 is not always possible. However, if all the factors are identical, then this remains possible, as is in the case where $\Omega$ is a polydisk $\Pi \cong D^{r}$. In this section we will deal with the case of polydisks. Then, for $w \in U \cap D$ we have $f^{\prime}(w)=\left(f_{1}^{\prime}(w), \cdots, f_{r}^{\prime}(w)\right)$. The Kähler-Einstein metric $g$ on $\Pi$ is given by

$$
\left.g\right|_{\Pi}=\frac{2\left|d z^{1}\right|^{2}}{\left(1-\left|z_{1}\right|^{2}\right)^{2}}+\cdots+\frac{2\left|d z^{r}\right|^{2}}{\left(1-\left|z_{r}\right|^{2}\right)^{2}} .
$$


Thus,

$$
\left\|d f\left(\frac{\partial}{\partial w}\right)\right\|^{2}=\frac{2\left|f_{1}^{\prime}(w)\right|^{2}}{\left(1-\left|f_{1}(w)\right|^{2}\right)^{2}}+\cdots+\frac{2\left|f_{r}^{\prime}(w)\right|^{2}}{\left(1-\left|f_{r}(w)\right|^{2}\right)^{2}} .
$$

In what follows we will write $f^{\prime}(w)$ to mean the tangent vector $d f\left(\frac{\partial}{\partial w}\right)$ at the point $w \in U$. The $k$-th term on the right-hand side of (10) is given by

$$
\frac{2\left|f_{k}^{\prime}(w)\right|^{2}}{\left(1-\left|f_{k}(w)\right|^{2}\right)^{2}}=\frac{\partial^{2}}{\partial w \partial \bar{w}}\left(-2 \log \left(1-\left|f_{k}\right|^{2}\right)\right)
$$

From $S=f(U \cap D) \subset \Pi \subset \Omega$ and $f(U \cap \partial D) \subset \partial \Pi$ and $f(b)=(1,0, \cdots, 0)$ it follows readily, shrinking the neighborhood $U$ of $b$ if necessary, we have $f_{1}(U) \cap$ $\partial D \subset \partial \Omega$ while $f_{k}(U \cap \bar{D}) \Subset D$. Hence $1-\left|f_{1}\right|^{2}=\left(1-|w|^{2}\right) s$ for some smooth function $s$ defined on $U$, hence the potential function $-2 \log \left(1-\left|f_{k}\right|^{2}\right)$ equals $-\log \left(1-\left|z_{1}\right|^{2}\right)+\log s$ while for $2 \leq k \leq r$ the potential function $-2 \log \left(1-\left|f_{k}\right|^{2}\right)$ is a smooth function defined on $U$. It follows from (8) that

$$
\frac{2\left|f_{1}^{\prime}(w)\right|^{2}}{\left(1-\left|f_{1}(w)\right|^{2}\right)^{2}}=\frac{2}{\left(1-|w|^{2}\right)^{2}}+O(1) ; \quad \frac{2\left|f_{k}^{\prime}(w)\right|^{2}}{\left(1-\left|f_{k}(w)\right|^{2}\right)^{2}}=O(1) \quad \text { for } \quad 2 \leq k \leq r
$$

on a neighborhood of $b$. Hence, for $w \in U \cap D$, the tangent vector $f^{\prime}(w)$ is equivalent under the action of $\operatorname{Aut}(\Pi)$ to a vector $\left(\xi_{1}(w), \cdots, \xi_{r}(w)\right) \in T_{0}(\Pi)$ where

$$
\left|\xi_{1}(w)\right|^{2}=\frac{1}{2 \delta(w)^{2}}+O(1) ; \quad\left|\xi_{k}(w)\right|^{2}=O(1) \quad \text { for } \quad 2 \leq k \leq r
$$

It follows that the tangent vector $f^{\prime}(w)$ is equivalent under $\operatorname{Aut}(\Pi)$ to a non-zero multiple of the tangent vector $\frac{1}{\sqrt{2}}\left(\mu_{1}(w), \cdots, \mu_{r}(w)\right) \in T_{0}(\Pi)$, where $\mu_{k}$ are real and nonnegative for $1 \leq k \leq n$, and furthermore

$$
\mu_{1}(w)=1+O(\delta(w)), \quad \mu_{k}(w)=O(\delta(w))
$$

(Here in $\mu_{1}(w)=1+O(\delta(w))$ the term $O(\delta(w))$ is necessarily nonpositive, but the sign of the term is irrelevant to us.) It follows that, in the notation used in formulating the condition $(\dagger)$ in the above, we have

$$
R_{\eta(w) \overline{\eta(w)} \eta(w) \overline{\eta(w)}}=-1+O\left(\delta(w)^{2}\right)
$$


verifying $(\dagger)$ in the special case where $S=f(U \cap D)$ lies on a maximal polydisk.

(3.4) Proof of Main Theorem in the general case For the general case of the proof of Main Theorem, we will instead verify $(\dagger)^{\prime}$, which implies $(\dagger)$, as explained in the above. We note that in the very special case where $f(U \cap D)$ lies on a maximal polydisk above, (12) implies that $(\dagger)^{\prime}$ is satisfied.

For the general case we first make some observations. By Lemma 3, every irreducible bounded symmetric domain $\Omega_{0}$ can be embedded as a totally geodesic complex submanifold of an irreducible bounded symmetric domain $\Omega=G_{0} / K$ where $G_{0}$ is a noncompact real form of a Lie group of type $A, D$ or $E$, so that all roots of $\mathfrak{g}$ are of equal length. From now on we assume for notational simplicity that $\Omega$ is irreducible, and for the proof of Main Theorem in the irreducible case without loss of generality we will assume that $\Omega=G_{0} / K$ is of type $A, D$ or $E$, in which case, fixing a Cartan subalgebra $\mathfrak{h} \subset \mathfrak{k}$, every noncompact positive root $\rho \in \Delta_{0}^{+}$is necessarily a minimal rational tangent. Choose now an orthonormal basis of $T_{0}(\Omega)$ consisting of roots of unit length belonging to noncompact positive roots $\rho \in \Delta_{0}^{+}$, and use Euclidean coordinates $\left(z_{1}, \cdots, z_{N}\right)$ on $\mathbb{C}^{N}$ arising from such a choice of an orthonormal basis $\left\{e_{\rho}: \rho \in \Delta_{0}^{+}\right\}, \Omega \subset \mathbb{C}^{N} \cong T_{0}(\Omega)$. As explained, for the proof of Main Theorem without loss of generality we may assume that $f(b)=(1,0, \cdots, 0)=e_{1}$. For $w \in U \cap D$ we can make use of the Polydisk Theorem to find a maximal polydisk $\Pi_{w} \subset \Omega$ passing through 0 and containing the point $f(w)$. ( $\Pi_{w} \subset \Omega$ is not necessarily unique.) However, the tangent vector $f^{\prime}(w) \in T_{f(w)}(\Omega)$ is not necessarily tangent to $\Pi_{w}$, and, to verify the condition $(\dagger)^{\prime}$ it is necessary also to consider the component of the tangent vector $f^{\prime}(w)$ normal to $\Pi_{w}$. As $w$ approaches $b \in U \cap D$ for the purpose of doing estimates we will slightly modify the procedure. We will move the point $f(w)$ to the minimal disk $D_{0}=\mathbb{C} e_{1} \cap \Omega$ on which metric calculations are easier, by Lemma 5.

If we identify $\Omega$ naturally as an open subset of $T_{0}(\Omega) \cong \mathbb{C}^{N}$, then, for $w \in U \cap D, f(w) \in \Omega$ is both a point on the bounded symmetric domain $\Omega$ and a vector on $T_{0}(\Omega)$. The vector $\eta(w):=f^{\prime}(w)$, which is a tangent vector of type $(1,0)$ at $f(w)$, will then be referred to as the velocity vector at $w$. Recall the maximal polydisk $\Pi \subset \Omega$ given by $\Pi=\left(\mathfrak{g}_{\psi_{1}} \oplus \cdots \mathfrak{g}_{\psi_{r}}\right) \cap \Omega$, where $\Psi=\left\{\psi_{1}, \cdots, \psi_{r}\right\}$ is a maximal strongly orthogonal set of positive noncompact roots and $\psi_{1}=\mu$ is the 
highest root. By the Polydisk Theorem, given any $w \in U \cap D$, there exists some $\gamma=\gamma_{w} \in K$ such that $\gamma(f(w))=\left(a_{1}(w), \cdots, a_{r}(w)\right) \in \Pi$, where $a_{k}(w)$ is real and nonnegative, and $1 \geq a_{1}(w) \geq a_{2}(w) \geq \cdots \geq a_{r}(w) \geq 0$. With the latter specifications each $a_{k}(w), 1 \leq k \leq r$, is uniquely determined.

Suppose $w_{i} \in U \cap D, 1 \leq i<\infty$, is a sequence converging to $b$ and we write $\gamma_{i}$ for $\gamma_{w_{i}}$. Then, $\gamma_{i}\left(f\left(w_{i}\right)\right)=\left(a_{1}\left(w_{i}\right), \cdots, a_{r}\left(w_{i}\right)\right)$. We write $\mathbb{N}^{+}=\{1,2, \cdots$, for the set of positive natural numbers. From the compactness of $K$ and the boundedness of $\left\{\left(a_{1}\left(w_{i}\right), \cdots, a_{r}\left(w_{i}\right)\right): 1 \leq i<\infty\right\}$ it follows that for some increasing function $\tau: \mathbb{N}^{+} \rightarrow \mathbb{N}^{+}$, the subsequence $\gamma_{\tau(i)}$ of $\gamma_{i}$ converges to some $\gamma_{\infty} \in K$ and the subsequence $\left(a_{1}\left(w_{\tau(i))}, \cdots, a_{r}\left(w_{\tau(i)}\right)\right)\right.$ of $\left(a_{1}\left(w_{i}\right), \cdots, a_{r}\left(w_{i}\right)\right)$ converges to some $p \in \bar{\Pi}$, where $p=\left(c_{1}, \cdots, c_{r}\right), 1 \geq c_{1} \geq c_{2} \geq \cdots c_{r} \geq 0$, and we have $\gamma_{\infty}(f(b))=p$. But since $f(b)=(1,0, \cdots, 0)$ is already in normal form, and the normal form of a tangent vector $\xi \in T_{0}(\Omega)$ is uniquely determined, we must have $c_{1}=1$ and $c_{k}=0$ for $2 \leq k \leq r$. Thus $\gamma_{\tau(i)}$ converges to $(1,0, \cdots, 0)$. Since this holds true for any choice of $\tau: \mathbb{N}^{+} \rightarrow \mathbb{N}^{+}$for which both $\gamma_{\tau(i)}$ and $\left(a_{1}\left(w_{\tau(i)}\right), \cdots, a_{r}\left(w_{\tau(i)}\right)\right)$ are convergent, it follows that in fact the normal forms $\left(a_{1}(w), \cdots, a_{r}(w)\right)$ of $f(w)$ must converge to $(1,0, \cdots, 0)$ as $w$ converges to $b$. (Write $h: T_{0}(\Omega) \rightarrow \mathbb{R}^{r}$ for the mapping which assigns to any tangent vector $\xi \in T_{0}(\Omega)$ its normal form $h(\xi) \in \mathbb{R}^{r}$. The preceding elementary arguments show precisely that $h$ is continuous.)

We are now ready to proceed with the proof of Main Theorem.

Proof of Main Theorem. Let $\Omega \Subset \mathbb{C}^{N}$ be a bounded symmetric domain of rank $r$. For notational simplicity we will assume that $\Omega$ is irreducible. At the end of the proof we will explain the minor changes that need to be made to carry the proof over to the reducible case. From the embedding $\Pi \subset \Omega$ we have naturally a monomorphism of Lie groups $\Phi: \operatorname{Aut}_{0}(\Pi) \rightarrow \operatorname{Aut}_{0}(\Omega)=G_{0}$. In what follows for any $\lambda \in \operatorname{Aut}_{0}(\Pi) \cong \operatorname{Aut}(D)^{r}$ the same symbol $\lambda$ will also be used to denote $\Phi(\lambda) \in G_{0}$. For $w \in U \cap D$ let $\lambda=\lambda_{w}$ be defined by

$$
\lambda\left(z_{1}, \cdots, z_{r}\right)=\left(z_{1}, \frac{z_{2}-a_{2}(w)}{1-a_{2}(w) z_{2}}, \cdots, \frac{z_{2}-a_{2}(w)}{1-a_{2}(w) z_{r}}\right)
$$

For $w_{0} \in U \cap D$ consider $h_{w_{0}}: U \cap D \rightarrow \Omega$ defined by

$$
h_{w_{0}}(w)=\lambda_{w_{0}}\left(\gamma_{w_{0}}(f(w))\right) \text {. }
$$


Then,

$$
\begin{gathered}
h_{w_{0}}\left(w_{0}\right)=\left(a_{1}(w), 0, \cdots, 0\right) \in \Pi ; \\
h_{w_{0}}^{\prime}(w)=d \lambda_{w_{0}}\left(\left(\gamma _ { w _ { 0 } } ( f ( w ) ) \left(\left(\gamma_{w_{0}}\left(f^{\prime}(w)\right)\right) .\right.\right.\right.
\end{gathered}
$$

Here $\left(\gamma_{w_{0}}(f(w))=\gamma_{w_{0}}(f(w))\right.$ is a point on $\Omega$ while $\gamma_{w_{0}}\left(f^{\prime}(w)\right)=\left(\gamma_{w_{0}} \circ f\right)^{\prime}(w)$ is a tangent vector at $\left(\gamma_{w_{0}}(f(w))\right.$. Denote by $\langle\cdot, \cdot\rangle_{\mathfrak{e}}$ the Euclidean inner product on $\mathbb{C}^{N}$ and by $\|\cdot\|_{\mathfrak{e}}$ the Euclidean norm. The mapping $f: U \cap D \rightarrow \mathbb{C}^{N}$ verifies the transversality condition at $b$ as is given in Lemma 7 , which, under the normalization $f(b)=(1,0, \cdots, 0)$, is given by $\left\langle f(b), \overline{f^{\prime}(b)}\right\rangle_{\mathfrak{e}} \neq 0$, which is equivalently $f_{1}^{\prime}(b) \neq 0$. Since $\gamma_{w_{0}} \in K$ is a unitary transformation, we have

$$
\left\langle\gamma_{w_{0}}(f(w)), \overline{\gamma_{w_{0}}\left(f^{\prime}(w)\right)}\right\rangle_{\mathfrak{e}}=\left\langle f(w), \overline{f^{\prime}(w)}\right\rangle_{\mathfrak{e}},
$$

which is equal to $\overline{f_{1}^{\prime}(b)} \neq 0$ at $w=b$. We have

$$
\left\langle h_{w_{0}}(w), \overline{h_{w_{0}}^{\prime}(w)}\right\rangle_{\mathfrak{e}}=\left\langle\lambda_{w_{0}}\left(\gamma_{w_{0}}(f(w)), \overline{d \lambda_{w_{0}}\left(( \gamma _ { w _ { 0 } } ( f ( w ) ) ) \left(\left(\gamma_{w_{0}}\left(f^{\prime}(w)\right)\right)\right.\right.}\right\rangle_{\mathfrak{e}}\right.
$$

Since $\lambda_{w_{0}}$, when regarded as automorphisms of $\Omega$, converges to the identity map $\operatorname{id}_{\Omega}$ in the Lie group $G_{0}$, shrinking $U$ if necessary there exists some open neighborhood $\Omega^{\sharp} \Subset \mathbb{C}^{N}$ of $\bar{\Omega}$ such that $\lambda_{w_{0}}$ is defined and holomorphic on $\Omega^{\sharp}$ whenever $w_{0} \in U$, and such that $d \lambda_{w_{0}}(w)$ converges uniformly on $\Omega^{\sharp}$ to the identity map on $T_{\Omega^{\sharp}}$ as $w_{0}$ converges to $b$. We may also assume without loss of generality that

$$
\left|\left\langle f(w), \overline{f^{\prime}(w)}\right\rangle_{\mathfrak{e}}\right|>\frac{1}{2}\left|f_{1}^{\prime}(b)\right|>0 \quad \text { for all } w \in U
$$

Given any $\epsilon>0$, shrinking $U$ further if necessary, we may assume that

$$
\left\|d \lambda_{w_{0}}^{\prime}(w)(\eta)-\eta\right\|_{\mathfrak{e}}<\epsilon\|\eta\|_{\mathfrak{e}}
$$

for every $w_{0} \in U \cap D$, every $w \in \Omega^{\prime}$ and every $\eta \in T_{w}\left(\Omega^{\prime}\right)$. It follows that

$$
\left\langle\lambda_{w_{0}}\left(\gamma_{w_{0}}(f(w)), \overline{d \lambda_{w_{0}}\left(\gamma_{w_{0}}(f(w))\right)\left(\gamma_{w_{0}}\left(f^{\prime}(w)\right)\right.}\right\rangle_{\mathfrak{e}}-\left\langle\gamma_{w_{0}}(f(w)), \overline{\gamma_{w_{0}}\left(f^{\prime}(w)\right)}\right\rangle_{\mathfrak{e}} \rightarrow 0\right.
$$


uniformly in $w \in U$ as $w_{0} \in U \cap D$ approaches $b$. It follows from (20), (22) and (24) that, shrinking the open neighborhood $U$ of $b$ further if necessary and substituting at $w=w_{0}$ we have

$$
\left|\left\langle h_{w_{0}}\left(w_{0}\right), \overline{h_{w_{0}}^{\prime}\left(w_{0}\right)}\right\rangle_{\mathfrak{e}}\right|>\frac{1}{4}\left|f_{1}^{\prime}(b)\right|>0
$$

for every $w_{0} \in U \cap D$.

Finally we combine (18) and (25) to get an estimate on the normal form of a vector $\eta_{w} \in T_{0}(\Omega)$ of unit length with respect to $g$ which is equivalent under the action of the holomorphic isometry group $G_{0}$ to the velocity vector $h_{w}^{\prime}(w) \in$ $T_{h_{w}(w)}(\Omega)$ as $w$ approaches $b$. Here by $(18)$, we have $h_{w}(w)=\left(a_{1}(w), 0, \cdots, 0\right)$, which lies on the minimal disk $D_{0}$, which allows us by Lemma 5 to describe the metric tensor $\left(g_{i \bar{j}}\right)\left(a_{1}(w), 0, \cdots, 0\right)$. Since $h_{w}^{\prime}(w)$ is uniformly bounded in the Euclidean norm for $w \in U \cap D$, we conclude from (25) and Lemma 5 that $h_{w}^{\prime}(w)$ is equivalent under $G_{0}$ to a non-zero multiple of a vector $\xi_{w} \in T_{0}(\Omega)$ of the form

$$
\begin{gathered}
\xi_{w}=\bigoplus_{\rho \in \Delta_{0}^{+}} a_{\rho} e_{\rho} ; \quad \text { where } a_{\mu}=\frac{1}{\delta(w)}, \\
a_{\rho}=O\left(\frac{1}{\sqrt{\delta(w)}}\right) \quad \text { whenever } \quad \mu-\rho \in D, \quad \text { and } \\
a_{\tau}=O(1) \quad \text { whenever } \tau \neq \mu \text { and } \mu-\tau \notin D .
\end{gathered}
$$

For the case where $\Omega$ is irreducible to prove Main Theorem it remains to deduce $(\dagger)^{\prime}$ from (26). In the notation of Lemma $2, \mathfrak{H}$ stands for the set of all noncompact positive roots $\rho$ such that $\mu-\rho$ is a root, and $\mathfrak{N}$ stands for the set of all noncompact positive roots $\tau$ such that $\rho \neq \mu$ and $\mu-\tau$ is not a root. Write now $\mathcal{H}:=\bigoplus\left\{\mathfrak{g}_{\rho}: \rho \in \mathfrak{H}\right\}$, and $\mathcal{N}:=\bigoplus\left\{\mathfrak{g}_{\rho}: \rho \in \mathfrak{N}\right\}$. We have $T_{0}(\Omega)=\mathbb{C} e_{\mu} \oplus \mathcal{H} \oplus \mathcal{N}$. From (26) we deduce that

$$
\eta_{w}=\frac{1}{\sqrt{1+O(\delta(w))}}\left(e_{\mu}+\bigoplus_{\rho \in \mathfrak{H}}\left(O(\sqrt{\delta(w)}) e_{\rho}+\bigoplus_{\tau \in \mathfrak{N}} O(\delta(w)) e_{\tau}\right)\right.
$$

It follows already from $(27)$ that $\eta_{w}$ converges to the minimal rational tangent $e_{\mu}=\alpha$ at $0 \in \Omega$ as $w$ approaches $b$. 
Recall the inclusions $\Omega \Subset \mathbb{C}^{N} \subset M$ which incorporate the Harish-Chandra realization $\Omega \Subset \mathbb{C}^{N}$ and the Borel embedding $\Omega \subset M$ of the bounded symmetric domain $\Omega$. Let $\pi_{0}: T_{0}(\Omega)-\{0\} \rightarrow \mathbb{P} T_{0}(\Omega)$ be the natural projection. For a subvariety $E \subset \mathbb{P} T_{0}(\Omega)$ we write $\widetilde{E}$ for its affinization $\pi^{-1}(E)$. Consider now the variety of minimal rational tangents $\mathcal{C}_{0} \subset \mathbb{P} T_{0}(M)=\mathbb{P} T_{0}(\Omega) . \mathcal{C}_{0}$ agrees with the highest weight orbit $\mathcal{W}_{0} \subset \mathbb{P} T_{0}(M)$ of the isotropy representation of $K^{\mathbb{C}}$ (cf. Hwang-Mok [HM, p.360ff.]). On the other hand, given a highest weight $\mu \in \Delta_{0}^{+}$, for $\alpha=e_{\mu} \in \widetilde{\mathcal{W}}_{0}$, writing $\widetilde{\mathcal{C}}_{0}=\widetilde{\mathcal{W}}_{0}=K^{\mathbb{C}} \alpha$, by considering the differential of the map $\Phi: K^{\mathbb{C}} \rightarrow T_{0}(\Omega)$ given by $\Phi(\gamma)=d \gamma(\alpha)$ it follows readily that $T_{\alpha}\left(\widetilde{\mathcal{C}}_{0}\right)=\mathbb{C} \alpha+\mathcal{H}$. (Alternatively, the same statement can be deduced from the Grothendieck decomposition $\left.T_{M}\right|_{C} \cong \mathcal{O}(2) \oplus \mathcal{O}(1)^{p} \oplus \mathcal{O}^{q}$ of the tangent bundle $T_{M}$ over a minimal ration curve $C$ passing through 0 with $T_{0}(C)=\mathbb{C} \alpha$ (cf. [HM, loc. cit.]) and from the general fact that $T_{0}\left(\widetilde{\mathcal{C}}_{0}\right)=P_{\alpha}$, where $P_{\alpha}$ is the positive part of the Grothendieck decomposition of $\left.T(M)\right|_{C}$ at $0 \in C$ (cf. [HM, pp.224-225]).) Thus, in a neighborhood of $\alpha$, the complex manifold $\widetilde{\mathcal{C}}_{0}$ can be parametrized by $U_{\epsilon}:=\{\xi \in \mathbb{C} \alpha+\mathcal{H}:\|\xi\|<\epsilon\}$ for some $\epsilon>0$, given by $\varphi(\xi)=\xi+\zeta(\xi)$, where $\zeta(\xi)$ is orthogonal to $\mathbb{C} \alpha+\mathcal{H}$ (i.e., $\zeta(\xi) \in \mathcal{N}$ ) for every $\xi \in U_{\epsilon}$, and where $\varphi(\xi) \leq C\|\xi\|^{2}$ for some positive constant $C$ and for every $\xi \in U_{\epsilon}$. (Actually, $\varphi(\xi)$ is a quadratic map in $\xi$, thus defined on all of the tangent space $\mathbb{C} \alpha+\mathcal{H}$, cf. [HM, p.377], but we will not use the latter fact.). Given this, rewriting in (27) the unit vector $\eta_{w}$ as

$$
\eta_{w}=\frac{\alpha+\xi_{w}+\chi_{w}}{\sqrt{1+\left\|\xi_{w}\right\|^{2}+\left\|\chi_{w}\right\|^{2}}}
$$

where $\xi_{w} \in \mathcal{H}$ and $\chi_{w} \in \mathcal{N}$, we deduce from (27) that

$$
\eta_{w}=\frac{\left(\alpha+\xi_{w}+\varphi\left(\xi_{w}\right)\right)+\left(\chi_{w}-\varphi\left(\xi_{w}\right)\right)}{\sqrt{1+\left\|\xi_{w}\right\|^{2}+\left\|\chi_{w}\right\|^{2}}}:=\alpha_{w}+\frac{\chi_{w}-\varphi\left(\xi_{w}\right)}{\sqrt{1+\left\|\xi_{w}\right\|^{2}+\left\|\chi_{w}\right\|^{2}}}
$$

where $\alpha_{w} \in \widetilde{\mathcal{C}_{0}}$ from the definition of $\varphi$. Thus, $\eta_{w}=\alpha_{w}+\zeta_{w}$, where $\zeta_{w} \in \mathcal{N}$, and it follows from (27) that

$$
\begin{gathered}
\left\|\zeta_{w}\right\| \leq\left\|\chi_{w}\right\|+\left\|\varphi\left(\xi_{w}\right)\right\| \leq\left\|\chi_{w}\right\|+C\left\|\xi_{w}\right\|^{2} \\
=O(\delta(w))+(O(\sqrt{\delta(w)}))^{2}=O(\delta(w))
\end{gathered}
$$


From now on we write $\mathcal{H}=\mathcal{H}_{\alpha}$ and $\mathcal{N}=\mathcal{N}_{\alpha}$. From the discussion above it follows that $\mathcal{N}_{\alpha}=(\mathbb{C} \alpha+\mathcal{H})^{\perp}$, i.e., $\mathcal{N}_{\alpha}$ is the orthogonal complement of $\mathbb{C} \alpha+\mathcal{H}_{\alpha}$ in $T_{0}(\Omega)$ with respect to the metric $g$, equivalently with respect to the Euclidean metric. The highest weight orbit $\mathcal{W} \subset \mathbb{P} T_{0}(\Omega)$ is in fact the $K$-orbit of $[\alpha]$ which turns out to be complex-analytic. (It is the unique complex-analytic $K$-orbit by the Borel-Weil Theorem). For any $\alpha^{\prime} \in \widetilde{\mathcal{C}}_{0}$, regarding $\alpha^{\prime}$ as a highest weight vector with respect to some choice of a Cartan subalgebra $\mathfrak{h}^{\prime} \subset \mathfrak{k}$, and we have likewise $T_{\alpha^{\prime}}\left(\widetilde{\mathcal{C}_{0}}\right)=\mathbb{C} \alpha^{\prime}+\mathcal{H}_{\alpha^{\prime}}$ and $\mathcal{N}_{\alpha^{\prime}}=\left(\mathbb{C} \alpha^{\prime}+\mathcal{H}_{\alpha^{\prime}}\right)^{\perp}$, where $\mathcal{H}_{\alpha^{\prime}}$ resp. $\mathcal{N}_{\alpha^{\prime}}$ is defined in analogy to $\mathcal{H}_{\alpha}$ resp. $\mathcal{N}_{\alpha}$.

From (30) we have $\eta_{w}=\alpha_{w}+\zeta_{w}$, where $\alpha_{w} \in \widetilde{\mathcal{C}}_{0}, \zeta_{w} \in \mathcal{N}_{\alpha}$, and $\left\|\zeta_{w}\right\|=$ $O(\delta(w))$. However, to verify $(\dagger)^{\prime}$ we have to prove instead $\eta_{w}=\alpha_{w}^{\prime}+\zeta_{w}^{\prime}$ where $\alpha_{w}^{\prime} \in \widetilde{\mathcal{C}}_{0}, \zeta_{w}^{\prime} \in \mathcal{N}_{\alpha_{w}^{\prime}}$ and $\left\|\zeta_{w}^{\prime}\right\|=O(\delta(w))$. We claim that this is indeed the case. To this end denote by $p: \mathbf{N} \rightarrow \widetilde{\mathcal{C}_{0}}$ the (smooth) normal bundle of $\widetilde{\mathcal{C}_{0}}$ as a Riemannian submanifold of $T_{0}(\Omega)-\{0\}$, Thus, over $\beta \in \widetilde{\mathcal{C}_{0}}$ we have $\mathbf{N}_{\beta}=$ $T_{\beta}\left(\widetilde{\mathcal{C}}_{0}\right)^{\perp}=\mathcal{N}_{\beta}$. Thus, $\mathbf{N} \subset \widetilde{\mathcal{C}_{0}} \times T_{0}(\Omega)$ by definition. The canonical projection of $\widetilde{\mathcal{C}_{0}} \times T_{0}(\Omega)$ onto the second factor $T_{0}(\Omega)$ restricts to a mapping on $\mathbf{N}$ which we denote by $q: \mathbf{N} \rightarrow T_{0}(\Omega)$. Consider the mapping $\Phi: \mathbf{N} \rightarrow T_{0}(\Omega)$ defined by $\Phi(\mathbf{n})=p(\mathbf{n})+q(\mathbf{n})$. Clearly, $\Phi$ is an open embedding of some neighborhood $\mathbf{U}$ of the zero section $Z \cong \widetilde{\mathcal{C}}_{0}$ in $\mathbf{N}$ onto a neighborhood $\mathcal{U}$ of $\widetilde{\mathcal{C}}_{0}$ in $T_{0}(\Omega)$. $\Phi$ is indeed the exponential map of the normal bundle $\mathbf{N}$ in the sense of Riemannian geometry, and the open neighborhoods $\mathbf{U} \supset Z$ and $\mathcal{U} \supset \widetilde{\mathcal{C}}_{0}$ can be chosen such that, if $\chi \in \mathcal{U}, \mathbf{u} \in \mathbf{U}$ and $\chi=\Phi(\mathbf{u})$, then $p(\mathbf{u}) \in \widetilde{\mathcal{C}}_{0}$ is the unique point on $\widetilde{\mathcal{C}}_{0}$ closest to $\chi$. From now on $\Phi$ will stand for the diffeomorphism $\left.\Phi\right|_{\mathbf{U}}: \mathbf{U} \rightarrow \mathcal{U}$. Since $\eta_{w}$ is of unit length, when $\zeta_{w}$ is sufficiently small, using the exponential $\operatorname{map} \Phi$ we can write $\eta_{w}=\alpha_{w}+\zeta_{w}=\alpha_{w}^{\prime}+\zeta_{w}^{\prime}$, where $\alpha_{w}^{\prime}=p\left(\Phi^{-1}\left(\eta_{w}\right)\right) \in \widetilde{\mathcal{C}}_{0}$ and $\zeta_{w} \in \mathcal{N}_{\alpha_{w}^{\prime}}$. From $\eta_{w}=\alpha_{w}+\zeta_{w}$ we see that the Euclidean distance of $\eta_{w}$ to $\widetilde{\mathcal{C}}_{0}$ is of order $O(\delta(w))$. Since $\left\|\zeta_{w}^{\prime}\right\|$ realizes the latter minimal distance, we have also $\left\|\zeta_{w}^{\prime}\right\|=O(\delta(w))$. We have therefore verified $(\dagger)^{\prime}$ in the case where $\Omega$ is irreducible, and completed the proof of Main Theorem in the irreducible case.

Finally, we observe that the proof also applies to the case where $\Omega$ is reducible. Writing $\Omega=\Omega_{1} \times \cdots \times \Omega_{m}, m \geq 2$, for the decomposition of $\Omega$ into the Cartesian product of irreducible bounded symmetric domains $\Omega_{\ell}, 1 \leq \ell \leq m$, the nonsingular part $\operatorname{Reg}(\partial \Omega)$ of the boundary of $\Omega \Subset \mathbb{C}^{N}$ in its Harish-Chandra 
realization is given by the disjoint union $\operatorname{Reg}(\partial \Omega)=\left(\operatorname{Reg}\left(\partial \Omega_{1}\right) \times \Omega_{2} \times \cdots \times \Omega_{m}\right) \cup$ $\cdots \cup\left(\operatorname{Reg}\left(\Omega_{1}\right) \times \cdots \times \Omega_{m-1} \times \partial \Omega_{m}\right)$. In the notation as in the statement of Main Theorem, suppose $f: U \rightarrow \Omega$ is a holomorphic curve such that $f(U \cap D) \subset \Omega$ and $f(U \cap \partial D) \subset \partial \Omega$, and such that $f(b) \in \operatorname{Reg}(\partial \Omega)$ for a general point $b \in U \cap \partial \Omega$. Because of the decomposition of $\operatorname{Reg}(\partial \Omega)$ as given in the above, shrinking $U$ if necessary without loss of generality we may assume that $f(U \cap \partial D)$ lies on one of the $m$ connected components of $\operatorname{Reg}(\partial \Omega)$ as given in the above. Renaming and reshuffling the factor domains $\Omega_{k}, 1 \leq k \leq m$, we may assume $f(U \cap \partial D) \subset$ $\operatorname{Reg}\left(\partial \Omega_{1}\right) \times \Omega_{2} \times \cdots \times \Omega_{m}$. The arguments for the proof of Main Theorem carry over almost verbatim with the following minor modification, viz., we normalize the Kähler-Einstein metric so that the Gauss curvature of a minimal disk $D$ on $\Omega_{1}$ is of Gauss curvature -1 . The holomorphic map $f=\left(f_{1}, \cdots, f_{m}\right)$, where for $1 \leq k \leq m$, we have $f_{k}: U \rightarrow \Omega_{k}$. Focusing at points converging to a general point $b \in U$ where $f$ is an embedding on a neighborhood of $b$ and composing with an automorphism of $\Omega$ we may assume without loss of generality that $f(b)=((1,0, \ldots, 0) ; 0 ; \ldots ; 0)$. Then $f_{\ell}(w)=O(\delta(w))$ for $2 \leq \ell \leq m$. For the rest of the arguments, writing $f_{k}=\left(f_{k}^{1}, \ldots, f_{k}^{N_{k}}\right)$ with respect to the Euclidean coordinates on $\Omega_{k} \Subset \mathbb{C}^{N_{k}}$, we have $\left(f_{\ell}^{j}\right)^{\prime}(w)=O(1)$ for $2 \leq \ell \leq m$ and $1 \leq j \leq m_{\ell}$, from which it follows, in analogy to the case of the polydisk as given in (12), we have $d f_{\ell}\left(\frac{\partial}{\partial w}\right)=O(1)$ as $w$ approaches $b$. The arguments leading to the curvature formula $(\dagger)$ carry over when we apply the arguments for the irreducible case to the factor $f_{1}$ and the arguments for the case of the polydisk to the other components $f_{\ell}, 2 \leq \ell \leq m$. Here we note that in dealing with $f_{1}$, for the argument analogous to the reduction of $(\dagger)$ to $(\dagger)^{\prime}$ we need only to argue with the variety of minimal rational tangents $\mathcal{C}_{0}\left(\Omega_{1}\right)$ of $\Omega$. The other varieties of minimal rational tangents $\Omega_{\ell}, 1 \leq \ell \leq m$ are irrelevant. The proof of Main Theorem is complete.

\section{REMARKS}

In the statement of Main Theorem, in place of a canonical Kähler-Einstein metric we may take $g$ to be any Aut $(\Omega)$-invariant Kähler-Einstein metric $g$, i.e. $g=$ $\pi_{1}^{*} g_{1}+\cdots+\pi_{m}^{*} g_{m}$ where, for $1 \leq k \leq m, g_{k}$ is a canonical Kähler-Einstein metric on $\Omega_{k}$, which is uniquely determined up to a positive scalar constant, and where $\pi_{k}: \Omega \rightarrow \Omega_{k}$ are the canonical projections. The proof is identical. 
(3.5) Concluding Remarks One of the original motivations for proving Main Theorem was to study asymptotic behavior of holomorphic isometric embeddings of the Poincaré disk into bounded symmetric domains, whose graphs extend by Mok [Mo5] to affine-algebraic subvarieties. For such mappings Main Theorem implies that, in the case where the general boundary point is a regular point of $\partial \Omega$, the isometric embedding is necessarily asymptotically totally geodesic at a general boundary point, and $\|\sigma\|^{2}$ must vanish precisely to the order 2 , i.e., it is of the first kind in the terminology of Mok [Mo4].

Another motivation was the study of holomorphic measure-preserving maps from an irreducible bounded symmetric domain to a Cartesian product of the same domain, in the sense of Clozel-Ullmo [CU], to which we refer the reader for definitions, in relation to the determination of commutants of certain modular correspondences of quotients $X:=\Omega / \Gamma$ of irreducible bounded symmetric domains by torsion-free lattices. In [CU] the authors derived germs of holomorphic measure-preserving maps from such commutants and reduced the problem essentially to a problem in complex differential geometry. The latter problem was solved in the case of the unit disk $\Omega=D$ in [CU], in the case of higherdimensional complex unit balls $\Omega=\mathbb{B}^{n}, n \geq 2$, in Mok [Mo3, 5] and in the case where $\Omega$ is of rank $\geq 2$ by Mok-Ng [MN2]. As a consequence, the following result (Theorem 1.1.2 of Mok-Ng [MN2]) on holomorphic measure-preserving maps was established.

Theorem (Mok-Ng [MN2]). Let $\Omega \Subset \mathbb{C}^{n}$ be an irreducible bounded symmetric domain, and $\Gamma \subset \operatorname{Aut}(\Omega)$ be a torsion-free lattice. Write $X:=\Omega / \Gamma$ and let $Y \subset X \times X$ be a measure-preserving algebraic correspondence with respect to the canonical measure $d \mu_{\Omega}$ on $\Omega$. Then, $Y$ is necessarily a modular correspondence.

In the case of $\Omega=\mathbb{B}^{n}, n \geq 2$, a stronger statement was established in Mok [Mo3] using Alexander's Theorem, where we actually proved that the graph of the germ of a holomorphic measure-preserving map $f=\left(f_{1}, \cdots, f_{p}\right):(\Omega ; 0) \rightarrow$ $(\Omega ; 0) \times \cdots \times(\Omega ; 0)$, where each $f_{k}$ is of maximal rank for $1 \leq k \leq p$, is necessarily totally geodesic in $\Omega \times(\Omega \times \cdots \times \Omega)$. The same statement for $\Omega$ of rank $\geq 2$ was established in Mok-Ng [MN2] by means of an Alexander-type theorem, where a germ of holomorphic map on $\mathbb{C}^{N}$ at $b \in \operatorname{Reg}(\partial \Omega)$ mapping $\Omega$ into $\Omega$ and $\partial \Omega$ into 
$\partial \Omega$ was shown to be necessarily the restriction of an automorphism $\gamma \in \operatorname{Aut}(\Omega)$. On the other hand, in the case of the unit disk $D$, in which case a holomorphic measure-preserving map is nothing other than a holomorphic isometry (up to a normalizing constant), examples of Mok [Mo5] show that the same statement fails, and in Clozel-Ullmo [CU] the theorem above for $\Omega=D$ was established by proving that $\operatorname{Graph}(f)$ extends to an affine-algebraic variety in $\mathbb{C} \times \mathbb{C}^{p}$ and by making use of the underlying action of $\Gamma$ on $\Omega$. Main Theorem in the current article shows that for any irreducible bounded symmetric domain, the germ of map $f$ as in the above arising from commutants of certain modular correspondences must be asymptotically totally geodesic when restricted to minimal disks passing through $b$ if $b$ is chosen generically. (In the case of the unit disk $D$ one only requires the special and much simpler case of Main Theorem for polydisks as was given in (3.3).) Coupled with the underlying action of $\Gamma$ on $\Omega$ this shows that $f$ is necessarily itself totally geodesic, since the behavior of $f$ (after extension) on a fundamental domain of $\Omega$ is recaptured by the asymptotic behavior of $f$ as the base point $x \in \Omega$ approaches a general regular boundary point $b \in \operatorname{Reg}(\Omega)$ owing to $\Gamma$-equivariance. Main Theorem therefore gives a uniform proof of the above theorem without resorting to Alexander-type extension theorems.

\section{References}

[CU] Clozel, L. and Ullmo, E.: Modular correspondences and invariant measures. J. Reine Angew. Math. 558 (2003), 47-83.

[FK] Faraut, J. and Korányi, A. Function spaces and reproducing kernels on bounded symmetric domains. J. Funct. Anal. 88 (1990), 64-89.

[Hel] Helgason, S.: Differential Geometry, Lie Groups, and Symmetric Spaces, Academic Press, Orlando 1978.

[Her] Hermann, R.: Geometric aspects of potential theory in bounded symmetric domains, Math. Ann. 151 (1963), 143-149.

[HM] Hwang, J.-M. and Mok, N. Varieties of minimal rational tangents on uniruled manifolds, in Several Complex Variables, ed. M. Schneider and Y.-T. Siu, MSRI publications 37, Cambridge University Press, Cambridge 1999, pp.351-389.

[Mo1] Mok, N.: Uniqueness theorems of Hermitian metrics of seminegative curva- 
ture on locally symmetric spaces of negative Ricci curvature, Ann. Math. 125 (1987), 105-152.

[Mo2] Mok, N.: Metric Rigidity Theorems on Hermitian Locally Symmetric Manifolds, Series in Pure Mathematics Vol.6, World Scientific, Singapore-New Jersey-London-Hong Kong, 1989.

[Mo3] Mok, N.: Local holomorphic isometric embeddings arising from correspondences in the rank-1 case, in Contemporary Trends in Algebraic Geometry and Algebraic Topology, ed. S.-S. Chern, L. Fu and R. Hain, Nankai Tracts in Mathematics, Vol.5, World Scientific, New Jersey 2002, pp.155-166.

[Mo4] Mok, N.: On the asymptotic behavior of holomorphic isometries of the Poincaré disk into bounded symmetric domains, Special Volume in honor of Professor Wu Wenjun on the occasion of his 90th birthday, Acta Mathematica Scientia 29B(4) (2009), 881-902.

[Mo5] Mok, N.: Extension of germs of holomorphic isometries up to normalizing constants with respect to the Bergman metric, J. Eur. Math. Soc. 14 (2012), 1617-1656.

[MN1] Mok, N. and Ng, S.-C.: Second fundamental forms of holomorphic isometries of the Poincar disk into bounded symmetric domains and their boundary behavior along the unit circle, Sci. in China Ser. A: Math. 52 (2009), 2628-2646.

[MN2] Mok, N. and Ng, S.-C.: Germs of measure-preserving holomorphic maps from bounded symmetric domains to their Cartesian products, J. Reine Angew. Math. 669 (2012), 47-73.

[MZ] Mok, N. and Zhong, J.-Q.: Curvature characterization of compact Hermitian symmetric spaces, J. Diff. Geom. 23 (1986), 15-67.

[Wo] Wolf, J. A. Fine structure of Hermitian symmetric spaces, in Geometry of Symmetric Spaces, ed. Boothby-Weiss, Marcel-Dekker, New York, 1972, pp.271-357.

Ngaiming Mok

The University of Hong Kong

Pokfulam Road, Hong Kong

E-mail: nmok@hku.hk 